\title{
Optimism, Delay and (In)Efficiency in a Stochastic Model of Bargaining
}

\author{
Juan Ortner* \\ Boston University
}

September 10, 2012

\begin{abstract}
I study a bilateral bargaining game in which the size of the surplus follows a stochastic process and in which players might be optimistic about their bargaining power. Following Yildiz (2003), I model optimism by assuming that players have different beliefs about the recognition process. I show that the unique subgame perfect equilibrium of this game might involve inefficient delays. I also show that these inefficiencies disappear when players can make offers arbitrarily frequently.
\end{abstract}

JEL Classification Codes: C73, C78.

Keywords: Bargaining, Optimism, Stochastic games, Dynamic games.

*Address: Department of Economics, Boston University, 270 Bay State Road, Boston, MA 02215, United States. E-mail: jortner@bu.edu. 


\section{Introduction}

Experimental and field evidence shows that, when bargaining, people tend to form optimistic beliefs about how future uncertainty will be resolved (e.g. Babcock and Loewenstein, 1997). This evidence also shows that there is a positive correlation between the optimism of the parties and the probability that bargaining ends in an impasse. In other words, this evidence identifies optimism as a possible explanation for bargaining delays.

However, Yildiz (2003) showed that optimism by itself cannot cause delays in bargaining. Yildiz (2003) studied a complete information bilateral bargaining game a la Rubinstein (1982) in which players have optimistic beliefs about their future bargaining power. The main result of his paper is that agreement will always be immediate whenever optimism is persistent and the number of bargaining rounds is sufficiently large.

In this paper, I extend Yildiz's (2003) model by allowing the size of surplus that the players are bargaining over to follow a stochastic process. The game has an infinite horizon and players have optimistic beliefs about their bargaining power. The model in this paper can also be thought of as an extension of the stochastic bargaining model of Merlo and Wilson $(1995,1998)$, allowing agents to have different beliefs about the recognition process. One of the main goals of the current paper is to show that persistent optimism can lead to costly delays in this stochastic environment. ${ }^{1}$

The following is a description of this paper's model and an overview of its main results. Let $S$ be a finite set of states and let $P$ be a transition matrix over elements in $S$. At each period until players reach an agreement, nature selects a state $s$ according to the one period ahead distribution implied by $P$. The state $s$ determines the size of the surplus. After agents learn the state, one player is recognized to make an offer. Players may have optimistic beliefs about the recognition process. The recognized player can either make a feasible offer or pass. In the first case, the responder can either accept or reject the offer. If she accepts the proposal the game ends. If the proposer chooses to pass or if the responder rejects the current offer, the game moves on to the next bargaining period.

By adapting arguments in Merlo and Wilson (1995, 1998), I show that this game has a unique subgame perfect equilibrium. The unique equilibrium satisfies the following property: there exists a set of states $S^{a}$ such that agents come to an agreement in period $t$ if and only if $s_{t} \in S^{a}$. Therefore, whether there is agreement or not at any given period depends only

\footnotetext{
${ }^{1}$ Cripps (1998) also considers a bargaining game in which the size of the surplus follows a Markov process. However, there are no differences in beliefs in his model, and inefficiencies can only arise in equilibrium when the buyer and the seller have different discount factors.
} 
on the size of the surplus, and is independent of the proposer's identity that period.

Suppose an agent is endowed with a surplus whose size follows the Markov process above. At each period, the agent must decide whether to consume the surplus or to wait. This problem is an optimal stopping problem, whose solution is given by an optimal stopping region $S^{*}$. At every state in $S^{*}$ it is optimal for the agent to stop the process and consume the surplus, while at any other state it is optimal to wait. Say that the outcome of the stochastic bargaining game is efficient if the set of states $S^{a}$ at which there is agreement is equal to $S^{*}$. Merlo and Wilson (1998) showed that these two sets are identical when agents have common beliefs about the recognition process. In contrast, in this paper I show that $S^{a}$ is always a subset of $S^{*}$ when agents hold optimistic beliefs. Importantly, this set inclusion might be strict. That is, there might be states at which it is optimal to consume the surplus but at which players fail to reach an agreement, as the following example shows.

Example 1 Suppose the surplus can take two possible values, $h=1$ and $l<h$. Let $\delta<1$ be the common discount factor. State $h$ is an absorbing state, while $\operatorname{Pr}\left(s_{t+1}=l \mid s_{t}=l\right)=P_{l l}$. Both players believe that they will make offers next period with probability 1 (regardless of the state next period). In this setting it is always efficient to consume the surplus at state $h$. Moreover, consuming the surplus at state $l$ is efficient if and only if

$$
l \geq \delta\left(P_{l l} l+\left(1-P_{l l}\right)\right) \Longleftrightarrow l \geq \frac{\delta\left(1-P_{l l}\right)}{1-\delta P_{l l}} .
$$

Since $h$ is an absorbing state, the results in Yildiz (2003) imply that players will reach an agreement the fist time $s_{t}=h$ : the proposer will obtain a payoff of $1 /(1+\delta)$ and the responder will obtain $\delta /(1+\delta)$.

Let $V_{i}(l)$ be player $i$ 's payoff at state $l$ from delaying an agreement until the state reaches $h$ :

$$
V_{i}(l)=\delta\left(P_{l l} V_{i}(l)+\frac{1-P_{l l}}{1+\delta}\right) \Rightarrow V_{i}(l)=\frac{\delta\left(1-P_{l l}\right)}{(1+\delta)\left(1-\delta P_{l l}\right)} .
$$

With probability $P_{l l}$ the state will be $l$ next period, in which case player $i$ again gets $V_{i}(l)$. With probability $1-P_{l l}$ the state will be $h$ next period, in which case player $i$ expects to obtain $1 /(1+\delta)$ since she believes she will make offers with probability 1 . If $V_{1}(l)+V_{2}(l)>l$ players will delay at state $l$, since there is no agreement that can satisfy both players' expectations. Since $V_{1}(l)+V_{2}(l)>\delta\left(1-P_{l l}\right) /\left(1-\delta P_{l l}\right)$, there are values of $\delta, P_{l l}$ and $l$ for which it is efficient to consume the surplus at state $l$ but for which players delay at this state.

The intuition behind these inefficiencies is as follows. When players reach an agreement, 
the player who makes the offer extracts a non-informational rent. If the surplus is expected to grow in the near term, the non-informational rent that the proposer gets in the future might be large relative to the current size of the surplus. When players are optimistic about the recognition process, they both expect to extract this large non-informational rent with high probability. In this case, the sum of what the players expect to get from delaying might be larger than the size of the surplus today, thereby making it impossible for them to reach an agreement. As Example 1 shows, this might occur even at states at which it is optimal to consume the surplus right away. The ultimate reason for this is that optimism about bargaining power has more impact in this stochastic environment, since now players can be optimistic about their bargaining power at states at which the surplus is large.

Example 1 shows how inefficient delays can arise when optimistic players bargain over a stochastic surplus. However, this paper also shows that these inefficiencies can only occur when there are non-negligible frictions in the bargaining process, and that they disappear when players can make offers arbitrarily frequently. The intuition behind this result is as follows. As the time between bargaining rounds goes to zero, the non-informational rent that the proposer gets also vanishes to zero: in the continuous-time limit the right to make an offer becomes extremely transient, so players get the same share of the surplus when they are making offers and when they are not. Therefore, when offers are arbitrarily frequently, the differences in beliefs about the recognition process no longer have an effect on the sum of the players expected continuation payoffs from delaying, and the outcome becomes fully efficient. The following example illustrates this.

Example 2 (Example 1 continued) Suppose that the surplus can take two possible values, $h=1$ and $l<h$. Let $\Delta$ measure the time between offers, with $\delta^{\Delta}=e^{-r \Delta}$ the discount factor. Suppose $h$ is an absorbing state, while $\operatorname{Pr}\left(s_{t+\Delta}=l \mid s_{t}=l\right)=e^{-\lambda \Delta}$ for some $\lambda \in(0, \infty)$. Both players believe that they will make offers next period with probability 1 . By equation (1), it is efficient to consume the surplus at state $l$ if and only if

$$
l \geq \frac{e^{-r \Delta}\left(1-e^{-\lambda \Delta}\right)}{1-e^{-r \Delta} e^{-\lambda \Delta}} \underset{\text { as } \Delta \rightarrow 0}{\rightarrow} \frac{\lambda}{r+\lambda} .
$$

By equation (2), the payoff player $i$ gets by always delaying an agreement at state $l$ is

$$
V_{i}^{\Delta}(l)=\frac{e^{-r \Delta}\left(1-e^{-\lambda \Delta}\right) h}{\left(1+e^{-r \Delta}\right)\left(1-e^{-r \Delta} e^{-\lambda \Delta}\right)} \underset{\text { as } \Delta \rightarrow 0}{\rightarrow} \frac{1}{2} \frac{\lambda}{r+\lambda},
$$

so $V_{1}^{\Delta}(l)+V_{2}^{\Delta}(l) \rightarrow \frac{\lambda}{r+\lambda}$ as $\Delta \rightarrow 0$. In the continuous-time limit players will delay at state 
$l$ only if $\frac{\lambda}{r+\lambda}>l$; that is, only if it is inefficient to consume the surplus at state $l$.

Related literature: This paper relates primarily to the literature of bargaining with optimism, starting with Yildiz's (2003) immediate agreement result. In a different paper, Yildiz (2004) shows that optimism about bargaining power may produce inefficient delays if players update their beliefs as the game goes on. Ali (2006) shows that optimism may lead to disagreement and delays in multilateral bargaining settings. Simsek and Yildiz (2009) consider a model in which the player's bargaining power evolves as a stochastic process, and show how optimism can lead to delays when players expect bargaining power to become more durable at some future date. ${ }^{2}$

More broadly, this paper also relates to the literature on bargaining impasses. Delays in bargaining can arise when players have private information (Kennan and Wilson, 1993), when players can commit not to accept poor offers (Fershtman and Seidmann, 1993) or when players may receive new information while bargaining (Avery and Zemsky, 1994). Inefficiencies may also arise when players can build a reputation for being irrational (Abreu and Gul, 2000), when outside options are history-dependent (Compte and Jehiel, 2004) or when players have higher order uncertainty (Feinberg and Skrzypacz, 2005).

\section{Model}

Let $N=\{1,2\}$ be the set of players, with typical elements $i$ and $j$, and let $S$ be a finite set of states. The realization of the state $s \in S$ each period determines the size of the surplus that period: there exists a function $c: S \rightarrow \mathbb{R}_{+}$such that $c(s)$ is the size of the surplus when the state is $s$. Let $s_{t}$ denote the realization of the state at time $t$. For simplicity, I assume that the random variable that determines the state at time zero is degenerate, assigning probability one to $s_{0} \in S$. From period zero onwards the state evolves according to an exogenous timehomogeneous Markov process. Let $P$ denote the transition matrix over elements in $S$, and let $P_{s s^{\prime}}$ denote the probability of moving from $s$ to $s^{\prime}$. At each date $t>0$ until players reach an agreement, nature draws a state $s_{t} \in S$ according to the distribution $\left\{P_{s_{t-1} s}\right\}_{s \in S}$.

At each date $t=0,1,2, \ldots$ before players reach an agreement, and after the realization of state $s \in S$, one player is randomly recognized to make an offer. The recognized player can either make an offer $u \in C\left(s_{t}\right):=\left\{x \in \mathbb{R}_{+}^{2}: x_{1}+x_{2} \leq c\left(s_{t}\right)\right\}$ or pass. In the first case, the other player must choose to either accept or reject the offer. If she accepts the offer the game ends and players receive their payoffs. If the proposer chooses to pass or if the responder

\footnotetext{
${ }^{2}$ See Yildiz (2011) for a complete review of the literature on bargaining with optimism.
} 
rejects the current offer the game moves on to the next period. At period $t+1$ nature draws a state $s_{t+1} \in S$ according to the distribution $\left\{P_{s_{t} s}\right\}_{s \in S}$, and then a new player is recognized to make an offer. The process continues until a player accepts an offer, so there is no limit on the number of bargaining rounds. ${ }^{3}$ Let $\delta<1$ be the common discount factor.

The difference between the model I study in this paper and the one studied by Merlo and Wilson $(1995,1998)$ is that I allow agents to hold different beliefs about the recognition process. Let $p_{s}^{i}$ be the probability with which player $i$ believes she will be recognized to make an offer when the state is $s \in S$. I only consider the case in which players are optimistic, so $p_{s}^{1}+p_{s}^{2} \geq 1$ for all $s \in S$. If this inequality is strict for some $s$, then players hold divergent beliefs at $s$. Define $y(s):=p_{s}^{1}+p_{s}^{2}-1$ to be the level of optimism in state $s$, with $y(s) \geq 0$ for all $s \in S$. Note that the players' beliefs about the recognition process depend only on the state $s$, and are independent of time. This difference in beliefs is common knowledge.

Let $Z=S \times N$. The set $Z$ is an expanded set of states, with $z=(s, i) \in Z$ denoting the state in which the surplus size is $c(s)$ and agent $i$ makes offers. Given the transition matrix $P$ over elements of $S$ and given the players' beliefs about the recognition process, I can calculate for each player $i$ a transition matrix $\widetilde{P}_{i}$ over elements in $Z$. Since players may have different beliefs about the recognition process, it might be that $\widetilde{P}_{1} \neq \widetilde{P}_{2}$. Let $E_{i}[\cdot \mid z]$ denote the conditional expectation associated with transition matrix $\widetilde{P}_{i}$, and let $E[\cdot \mid s]$ denote the conditional expectation associated with transition matrix $P$.

An outcome of this bargaining game is a pair $(u, \tau)$, where $\tau$ is a stopping time and $u=\left(u_{1}, u_{2}\right)$ is a 2 -dimensional random variable, measurable with respect to the $\sigma$-algebra generated by $z^{\tau}=\left(z_{1}, \ldots, z_{\tau}\right)$, with $u\left(s_{\tau}, j_{\tau}\right) \in C\left(s_{\tau}\right)$ if $\tau<\infty$ and $u=0$ if $\tau=\infty$. That is, an outcome $(u, \tau)$ is given by a time $\tau$ at which players reach an agreement and a random variable $u$ which determines the share of the surplus that each agent gets. I assume that players are risk-neutral expected utility maximizers: given an initial state $z$, player $i$ 's payoff from outcome $(u, \tau)$ is given by $E_{i}\left[\delta^{\tau} u_{i}\left(z_{\tau}\right) \mid z\right]$.

An outcome $(u, \tau)$ is stationary if there exists a subset $Z^{a} \subseteq Z$ and a function $\eta: Z^{a} \rightarrow \mathbb{R}^{2}$ such that (i) $\tau:=\inf \left\{t \geq 0:\left(s_{t}, i_{t}\right) \in Z^{a}\right\}$ and (ii) $u\left(s_{\tau}, i_{\tau}\right)=\eta\left(s_{\tau}, i_{\tau}\right) \in C\left(s_{\tau}\right)$. That is, under a stationary outcome players reach an agreement the first time the state reaches the set $Z^{a}$, in which case the division of the surplus is $\eta\left(z_{\tau}\right)$. Given an initial state $z$, agent $i$ 's

\footnotetext{
${ }^{3}$ Although I assume that the game has an infinite horizon, the setup can incorporate games with a finite horizon or games with an exogenous risk of breakdown. To do this, I need to add an absorbing state $\widetilde{s}$ such that $c(\widetilde{s})=0$ (and in the case of a finite horizon I also need to expand the set of states $S$ accordingly, so that a state now represents the size of the surplus and the time left until the final bargaining round).
} 
utility from stationary outcome $(u, \tau)$ is

$$
V_{i}(z)= \begin{cases}\eta_{i}(z) & \text { if } z \in Z^{a} \\ E_{i}\left[\delta^{\tau} \eta_{i}\left(z_{\tau}\right) \mid z_{0}=z\right] & \text { if } z \notin Z^{a}\end{cases}
$$

In what follows, I will denote a stationary outcome by $\left(Z^{a}, \eta\right)$.

Finally, a stationary outcome $\left(Z^{a}, \eta\right)$ is anonymous if $Z^{a}=S^{a} \times N$ for some $S^{a} \subseteq S$. Thus, in an anonymous stationary outcome players reach an agreement the first time the Markov process enters the set $S^{a}$, independently of which player makes an offer that period. Given an initial state $(s, k)$, player $i$ 's payoff from outcome $\left(S^{a} \times N, \eta\right)$ is

$$
V_{i}(s, k)= \begin{cases}\eta_{i}(s, k) & \text { if } s \in S^{a}, \\ E\left[\delta^{\tau}\left(p_{s_{\tau}}^{i} \eta_{i}\left(s_{\tau}, i\right)+\left(1-p_{s_{\tau}}^{i}\right) \eta_{i}\left(s_{\tau}, i\right)\right) \mid s_{0}=s\right] & \text { if } s \notin S^{a}\end{cases}
$$

Note that in this case I can drop the subscripts in the expectations, since now the stopping time $\tau$ depends only on the realization of $s_{0}, s_{1}, s_{2}, \ldots$; i.e., in this case $\tau=\inf \left\{t \geq 0: s_{t} \in S^{a}\right\}$.

For each player, a strategy specifies a feasible action at every history at which that player must act. A strategy profile is a pair of strategies, one for each player. A strategy profile $\sigma$ induces an outcome $(u, \tau)$, and hence an expected payoff for each player at every history of the game. A strategy profile $\sigma$ is a subgame perfect equilibrium (SPE) if for every player $i \in N, \sigma_{i}$ is a best response to $\sigma_{-i}$ at every history.

\section{$3 \quad$ SPE payoffs}

In this section, I show that this bargaining game has unique SPE payoffs and that the game also has a unique SPE outcome. This unique SPE outcome is both stationary and anonymous. For any $f: S \rightarrow \mathbb{R}$, let $E_{s}\left[f\left(s^{\prime}\right)\right]=\sum_{s^{\prime} \in S} P_{s s^{\prime}} f\left(s^{\prime}\right)$ denote the expectation of $f\left(s_{t+1}\right)$ conditional on $s_{t}=s$.

Theorem 1 There exists unique SPE payoffs. For every $(s, k) \in Z$, let $V_{i}(s, k)$ denote the payoff of player $i$ when the state is $(s, k)$. These payoffs satisfy:

$$
\begin{aligned}
& V_{i}(s, j)=\delta E_{s}\left[p_{s^{\prime}}^{i} V_{i}\left(s^{\prime}, i\right)+\left(1-p_{s^{\prime}}^{i}\right) V_{i}\left(s^{\prime}, j\right)\right] \\
& V_{j}(s, j)=\max \left\{\begin{array}{c}
c(s)-\delta E_{s}\left[p_{s^{\prime}}^{i} V_{i}\left(s^{\prime}, i\right)+\left(1-p_{s^{\prime}}^{i}\right) V_{i}\left(s^{\prime}, j\right)\right], \\
\delta E_{s}\left[p_{s^{\prime}}^{j} V_{j}\left(s^{\prime}, j\right)+\left(1-p_{s^{\prime}}^{j}\right) V_{j}\left(s^{\prime}, i\right)\right]
\end{array}\right\} .
\end{aligned}
$$




\section{Proof. See Appendix A.1.}

Let $V: Z \rightarrow \mathbb{R}^{2}$ be the unique SPE payoffs. Suppose the state is $(s, j) \in Z$ and consider the equilibrium payoff of player $i \neq j$ at that state. If player $j$ chooses to pass or if player $j$ makes an offer that player $i$ rejects, then player $i$ 's utility at state $(s, j)$ is given by her continuation payoff. On the other hand, if player $j$ makes an offer that player $i$ accepts, this offer must give player $i$ her continuation payoff, since in equilibrium player $i$ will accept any offer that gives her this much. Therefore, the equilibrium payoff of player $i$ at state $(s, j)$ is given by her continuation value, regardless of whether there is agreement at that state or not; this is the content of equation (3).

Consider next the equilibrium payoff of player $j$ at state $(s, j)$. If player $j$ is to make an offer that player $i$ will accept, she will offer to give player $i$ her continuation payoff. In this case, player $j$ gets a payoff equal to the size of the surplus $c(s)$ minus the continuation payoff of player $i$. However, player $j$ can also choose to pass on her right to make an offer, in which case she gets her own continuation payoff. Therefore, player $j$ will make an offer that player $i$ will accept only if the first quantity is larger than the second, and will choose to pass otherwise; this is the content of equation (4).

It follows from equation (4) that players will reach an agreement at state $(s, j)$ only if

$$
c(s)-\delta E_{s}\left[p_{s^{\prime}}^{i} V_{i}\left(s^{\prime}, i\right)+\left(1-p_{s^{\prime}}^{i}\right) V_{i}\left(s^{\prime}, j\right)\right] \geq \delta E_{s}\left[p_{s^{\prime}}^{j} V_{j}\left(s^{\prime}, j\right)+\left(1-p_{s^{\prime}}^{j}\right) V_{j}\left(s^{\prime}, i\right)\right],
$$

and will delay if the reverse inequality holds. Moreover, since continuation values are independent of the player who is making offers, if there is agreement at state $(s, j)$ there will also be agreement at state $(s, i)$. If equation $(5)$ holds with equality then equilibrium is consistent with both agreement and delay at state $(s, j)$. From now on I will ignore this trivial source of multiplicity and assume that players always come to an agreement in state $(s, j)$ if the inequality in equation (5) holds with equality.

Define the function $\phi: S \rightarrow \mathbb{R}$ as

$$
\phi(s)=\delta E_{s}\left[p_{s^{\prime}}^{1} V_{1}\left(s^{\prime}, 1\right)+\left(1-p_{s^{\prime}}^{1}\right) V_{1}\left(s^{\prime}, 2\right)+p_{s^{\prime}}^{2} V_{2}\left(s^{\prime}, 2\right)+\left(1-p_{s^{\prime}}^{2}\right) V_{2}\left(s^{\prime}, 1\right)\right]
$$

Let $S^{a}:=\{s \in S: c(s) \geq \phi(s)\}$. It then follows that the set of states at which there is agreement is $S^{a} \times N$. That is, the SPE outcome of this stochastic bargaining game is stationary and anonymous.

Remark 1 In Appendix A.1, I show that the unique SPE payoffs in Theorem 1 can be 
derived via iterated conditional dominance (Proposition A1). Therefore, as in Yildiz (2003), the analysis in the paper is immune to the critique of Dekel, Fudenberg and Levine (2004) to games in which players have heterogeneous beliefs.

\section{Delay and inefficiency}

Let $w: S \rightarrow \mathbb{R}$ be the solution to

$$
w(s)=\sup _{\tau \in T} E\left[\delta^{\tau} c\left(s_{\tau}\right) \mid s_{0}=s\right],
$$

where $T$ is the set of stopping times. Problem (6) is an optimal stopping problem. This problem arises when one agent with discount factor $\delta$ has complete control over the process $c(s)$ and must decide at each time period whether to consume the surplus or wait. Using standard dynamic programming arguments one can show that $w$ is the unique solution to

$$
w(s)=\max \left\{c(s), \delta E_{s}\left[w\left(s^{\prime}\right)\right]\right\} .
$$

Moreover, the stopping time that solves problem (6) is given by $\tau^{*}=\inf \left\{t \geq 0: s_{t} \in S^{*}\right\}$, with $S^{*}:=\{s \in S: w(s)=c(s)\}$.

Definition 1 An anonymous stationary outcome $(\widetilde{S} \times N, \eta)$ is efficient if and only if $\widetilde{S}=S^{*}$.

Let $\left(S^{a} \times N, \eta\right)$ be the SPE outcome. The main result of this section shows that it is always the case that $S^{a} \subseteq S^{*}$. That is, the set of states at which there is agreement is a subset of the set of states at which it is optimal to stop the process and consume the surplus. This implies that the only type of inefficiency that can arise in equilibrium is that players wait for too long before coming to an agreement.

Let $V: Z \rightarrow \mathbb{R}^{2}$ be the unique SPE payoffs of the bargaining game. Equations (3) and (4) imply that $V_{1}(s, k)+V_{2}(s, k)=\max \{c(s), \phi(s)\}$ for every $s \in S$ and $k=1,2$. Since $V_{1}(s, 1)+V_{2}(s, 1)=V_{1}(s, 2)+V_{2}(s, 2)$ for all $s \in S$, it follows that

$$
\phi(s)=\delta E_{s}\left[V_{1}\left(s^{\prime}, 1\right)+V_{2}\left(s^{\prime}, 1\right)+y\left(s^{\prime}\right)\left(V_{1}\left(s^{\prime}, 1\right)-V_{1}\left(s^{\prime}, 2\right)\right)\right],
$$

where $y\left(s^{\prime}\right)=p_{s^{\prime}}^{1}+p_{s^{\prime}}^{2}-1$ for all $s^{\prime} \in S$. For all $s \in S$, let

$$
\widetilde{v}(s)=y(s)\left(V_{1}(s, 1)-V_{1}(s, 2)\right)=y(s)(c(s)-\phi(s)) \mathbf{1}_{\left\{s \in S^{a}\right\}},
$$


where $\mathbf{1}_{\{\cdot\}}$ denotes the indicator function. Note that $\widetilde{v}(s)=0$ for all $s \notin S^{a}$. Moreover, since $V_{1}(s, 1) \geq V_{1}(s, 2)$ for all $s \in S^{a}$ and since $y(s) \geq 0$ for all $s \in S$, it follows that $\widetilde{v}(s) \geq 0$ for all $s \in S$. Therefore, $\phi(s)=\delta E_{s}\left[V_{1}\left(s^{\prime}, k\right)+V_{2}\left(s^{\prime}, k\right)+\widetilde{v}\left(s^{\prime}\right)\right]$, and

$$
V_{1}(s, k)+V_{2}(s, k)=\max \left\{c(s), \delta E_{s}\left[V_{1}\left(s^{\prime}, k\right)+V_{2}\left(s^{\prime}, k\right)\right]+\delta E_{s}\left[\widetilde{v}\left(s^{\prime}\right)\right]\right\} .
$$

Let $W: S \rightarrow \mathbb{R}$ be given by $W(s)=V_{1}(s, k)+V_{2}(s, k)$. Equation (8) implies that

$$
W(s)=\max \left\{c(s), \delta E_{s}\left[W\left(s^{\prime}\right)\right]+\delta E_{s}\left[\widetilde{v}\left(s^{\prime}\right)\right]\right\} .
$$

Finally, note that $S^{a}=\{s \in S: \phi(s) \leq c(s)\}=\{s \in S: W(s)=c(s)\}$.

Theorem 2 Let $\left(S^{a} \times N, \eta\right)$ be the SPE outcome. Then $S^{a} \subseteq S^{*}$.

Proof. Recall that $S^{*}=\{s \in S: w(s)=c(s)\}$ and $S^{a}=\{s \in S: W(s)=c(s)\}$. To prove Theorem 2 it suffices to show that $W(s) \geq w(s)$ for all $s \in S$. To see that these inequalities hold, let $T^{\prime}: F(S) \rightarrow F(S)$ be the operator

$$
T^{\prime}(f)(s)=\max \left\{c(s), \delta E_{s}\left[\widetilde{v}\left(s^{\prime}\right)\right]+\delta E_{s}\left[f\left(s^{\prime}\right)\right]\right\} .
$$

Operator $T^{\prime}$ is a contraction with modulus $\delta$ and $T^{\prime}(W)=W$ is its unique fixed point.

The operator $T^{\prime}$ is increasing. That is, if $f, g \in F(S)$ are such that $f(s) \geq g(s)$ for all $s \in S$, then $T^{\prime}(f)(s) \geq T^{\prime}(g)(s)$ for all $s \in S$. To see this, note that there are two possible cases: (i) $T^{\prime}(g)(s)=c(s)$ and (ii) $T^{\prime}(g)(s)=\delta E_{s}\left[\widetilde{v}\left(s^{\prime}\right)\right]+\delta E_{s}\left[g\left(s^{\prime}\right)\right]$. In case (i), $T^{\prime}(f)(s) \geq c(s)=T^{\prime}(g)(s)$. In case (ii),

$$
T^{\prime}(f)(s) \geq \delta E_{s}\left[\widetilde{v}\left(s^{\prime}\right)\right]+\delta E_{s}\left[f\left(s^{\prime}\right)\right] \geq \delta E_{s}\left[\widetilde{v}\left(s^{\prime}\right)\right]+\delta E_{s}\left[g\left(s^{\prime}\right)\right]=T^{\prime}(g)(s),
$$

where the first inequality follows from the definition of $T^{\prime}$ and the second one from the assumption that $f(s) \geq g(s)$ for all $s \in S$. Define the sequence $\left\{f^{r}\right\}$ with $f^{1}=w$ and $f^{r}=T^{\prime}\left(f^{r-1}\right)$ for all $r>1$, where $w$ is the solution to $(7)$. Note that $f^{2}(s)=T^{\prime}\left(f^{1}\right)(s) \geq$ $f^{1}(s)=w(s)$ for all $s \in S$. Since $T^{\prime}$ is increasing, it follows by induction that $f^{r}(s) \geq$ $f^{r-1}(s)$ for all $s \in S$ and for all $r \geq 2$. Finally, the fact that $T^{\prime}$ is a contraction implies that $\left\{f^{r}\right\} \rightarrow W$. I conclude that $W(s) \geq w(s)$ for all $s \in S$, which in turn implies $S^{a} \subseteq S^{*}$.

Corollary 1 If players have common beliefs about the recognition process (so $y(s)=0$ for all $s \in S$ ), then $S^{a}=S^{*}$. 
Proof. Note that $y(s)=0$ for all $s \in S$ implies that $\widetilde{v}(s)=0$ for all $s \in S$. In this case equation (9) becomes

$$
W(s)=\max \left\{c(s), \delta E_{s}\left[W\left(s^{\prime}\right)\right]\right\} .
$$

Note that the solution to $\left(9^{\prime}\right)$ is equal to the solution to $(7)$, so $W(s)=w(s)$ for all $s \in S$. Thus, $S^{a}=\{s: W(s)=c(s)\}=\{s: w(s)=c(s)\}=S^{*}$.

Corollary 1 is a known result, which I include here only for completeness. Indeed, when $y(s)=0$ for all $s \in S$ the model in this paper becomes a special case of the stochastic bargaining game with transferable utility studied by Merlo and Wilson (1998), and they showed that in their model there can only be efficient delays. ${ }^{4}$

Theorem 2 and Corollary 1 highlight which are the sources of inefficiencies in this stochastic bargaining model with differences in beliefs. When agents have optimistic beliefs about the recognition process, the sum of what the players expect to get if they delay an agreement might be larger than the value of waiting in the single person problem; i.e., $W(s) \geq w(s)$. Therefore, there might be states at which it is optimal to stop the single person problem but at which there is no division of the surplus that satisfies both parties' expectations (as in Example 1 in the Introduction). In other words, players might wait for too long before reaching an agreement, leading to inefficient delays.

Remark 2 In this paper, I assume that players have optimistic beliefs about their bargaining power and common beliefs about the evolution of the stochastic process that drives the size of the surplus. The assumption of common beliefs about the stochastic process allows me to define efficiency in terms of the solution to the optimal stopping problem (6). One of the main insights of this paper is that optimism about bargaining power may lead to inefficient delays in this stochastic environment; i.e., that players may delay an agreement at states at which it would be optimal to consume the surplus. One could also allow players to have divergent beliefs about the evolution of the underlying stochastic process driving the surplus. However, in such a setting it would not be possible to make statements about the efficiency of the bargaining outcome without making assumptions regarding the structure of the "true" stochastic process governing the size of the surplus.

\footnotetext{
${ }^{4}$ The efficiency result in Merlo and Wilson (1998) depends crucially on the assumption that utility is perfectly transferable between players. Indeed, Merlo and Wilson (1995) show that inefficiencies may arise in stochastic bargaining games with non-transferable utility. In this setting players may reach an agreement at states at which it would be optimal to delay, since they are not able to commit to future divisions of the surplus.
} 


\subsection{Conditions for inefficiencies}

In this subsection, I study conditions under which inefficient delays will arise in the unique SPE. I start by analyzing a simple setting in which the surplus can take two possible values. Then, I consider the general setting with any finite number of states.

Suppose that there are two possible states, $S=\{h, l\}$, with $c(h)>c(l)$. In this setting, it is always efficient to consume the surplus at state $h$, so $h \in S^{*}$. It also true that $h \in S^{a}$, so players will always reach an agreement at state $h$. To see this, suppose by contradiction that $h \notin S^{a}$. Since $S^{a} \neq \emptyset$, it follows that $S^{a}=l .{ }^{5}$ Then, it must be that

$$
c(h)<W(h)=\delta\left(P_{h h} W(h)+\left(1-P_{h h}\right) c(l)\right)+\delta y(l)\left(1-P_{h h}\right)(c(l)-\phi(l)) .
$$

Since $\phi(l) \geq \delta c(l)$, it follows from (10) that

$$
W(h) \leq \frac{\delta\left(1-P_{h h}\right) c(l)(1+y(l)(1-\delta))}{1-\delta P_{h h}} \leq \frac{\delta\left(1-P_{h h}\right) c(l)(2-\delta)}{1-\delta P_{h h}}<c(l)<c(h)
$$

a contradiction. Thus, it must be that $h \in S^{a}$. This implies that inefficient delays can only arise in this setting if $S^{*}=\{h, l\}$; otherwise, $\{h\}=S^{*} \supseteq S^{a} \ni h$, so $S^{a}=S^{*}$.

Proposition 1 Suppose $S=\{h, l\}$, with $c(h)>c(l)$. Then, the unique SPE is inefficient if and only if

$$
\frac{\delta\left(1-P_{l l}\right)(1+y(h)) c(h)}{\left(1-\delta P_{l l}\right)\left(1+\delta y(h) P_{h h}\right)+\delta^{2}\left(1-P_{l l}\right) y(h)\left(1-P_{h h}\right)}>c(l) \geq \frac{\delta\left(1-P_{l l}\right) c(h)}{1-\delta P_{l l}} .
$$

Proof. Suppose that the SPE is inefficient. By the discussion above, it must be that $S^{*}=\{h, l\}$ and that $S^{a}=\{h\}$. The fact that $S^{*}=\{h, l\}$ implies

$$
c(l) \geq \frac{\delta\left(1-P_{l l}\right) c(h)}{1-\delta P_{l l}}
$$

On the other hand, $l \notin S^{a}$ implies

$$
\begin{aligned}
W(l) & =\delta\left(P_{l l} W(l)+\left(1-P_{l l}\right) c(h)\right)+\delta\left(1-P_{l l}\right) y(h)(c(h)-\phi(h))>c(l), \\
\phi(h) & =\delta\left(P_{h h} c(h)+\left(1-P_{h h}\right) W(l)\right)+\delta y(h) P_{h h}(c(h)-\phi(h)) .
\end{aligned}
$$

\footnotetext{
${ }^{5}$ If $S^{a}=\emptyset$ then the payoffs of both players would be zero, which cannot occur in a SPE: in this case the proposer could always offer her opponent a share $\varepsilon>0$ of the current surplus (an offer her opponent would accept) and earn $c(s)-\varepsilon$.
} 
Combining equations (12) and (13), it follows that

$$
W(l)=\frac{\delta\left(1-P_{l l}\right)(1+y(h)) c(h)}{\left(1-\delta P_{l l}\right)\left(1+\delta y(h) P_{h h}\right)+\delta^{2}\left(1-P_{l l}\right) y(h)\left(1-P_{h h}\right)}>c(l) .
$$

On the other hand, if (11) does not hold then either $S^{a}=S^{*}=\{h\}$ or $S^{a}=S^{*}=\{h, l\}$.

Proposition 1 provides necessary and sufficient conditions for inefficiencies in settings in which the surplus can take two possible values. From equation (11) it follows that inefficient delays can only arise whenever players are optimistic about their bargaining power at state $h$ : the inequalities in equation (11) can never hold if $y(h)=0$, since in this case the left hand side of (11) would be equal to the right hand side. That is, inefficiencies can only arise when players are optimistic about their bargaining power at the state at which the surplus is large. On the other hand, the conditions in (11) do not depend on $y(l)$, so whether or not there are inefficient delays in this setting is independent on the level of optimism at state $l$.

The intuition behind these results is as follows. When players reach an agreement at state $h$, the player who gets to be proposer extracts a non-informational rent: she obtains a larger payoff than what she would obtain if she was responder. If players are optimistic about their relative bargaining power at state $h$, then they both expect to extract this non-informational rent with high probability. In this case, the sum of what the players expect to get from delaying an agreement at state $l$ will be strictly larger than the value of waiting in the single person optimal stopping problem. Given this large perceived value of waiting, players might delay an agreement at state $l$ even in situations in which it would be efficient to agree. Note that the perceived value of waiting at state $l$ depends only on the level of optimism at state $h$ (the state at which players expect to extract the non-informational rent), and is independent of the level of optimism at state $l$. Thus, inefficiencies can only arise in this environment whenever $y(h)$ is large enough.

Consider next the general setting in which $S$ is any finite set of states. Let $\bar{c}=\max _{s \in S} c(s)$ and $\underline{c}=\min _{s \in S} c(s)$ denote the largest and smallest size of the surplus, respectively. Let $\bar{y}=\max _{s \in S} y(s)$ denote the highest level of optimism.

Proposition 2 Suppose there exists a state $s \in S^{*}$ such that

$$
\delta E_{s}\left[w\left(s^{\prime}\right)\left(1+y\left(s^{\prime}\right)\right)\right]-\delta^{2} E_{s}\left[y\left(s^{\prime}\right)\right](\bar{c}+\bar{y}(\bar{c}-\delta \underline{c}))>c(s)
$$

Then, $s \notin S^{a}$, so the SPE outcome of the bargaining game is inefficient. 
Proof. Suppose there exists a state $s \in S^{*}$ such that (14) holds. Note then that

$$
\begin{aligned}
\phi(s) & =\delta E_{s}\left[W\left(s^{\prime}\right)\right]+\delta E_{s}\left[y\left(s^{\prime}\right)\left(c\left(s^{\prime}\right)-\phi\left(s^{\prime}\right)\right) \mathbf{1}_{\left\{s^{\prime} \in S^{a}\right\}}\right] \\
& =\delta E_{s}\left[W\left(s^{\prime}\right)\right]+\delta E_{s}\left[y\left(s^{\prime}\right)\left(W\left(s^{\prime}\right)-\phi\left(s^{\prime}\right)\right)\right] \\
& \geq \delta E_{s}\left[W\left(s^{\prime}\right)\left(1+y\left(s^{\prime}\right)\right)\right]-\delta^{2} E_{s}\left[y\left(s^{\prime}\right)(\bar{c}+\bar{y}(\bar{c}-\delta \underline{c}))\right] \\
& \geq \delta E_{s}\left[w\left(s^{\prime}\right)\left(1+y\left(s^{\prime}\right)\right)\right]-\delta^{2} E_{s}\left[y\left(s^{\prime}\right)(\bar{c}+\bar{y}(\bar{c}-\delta \underline{c}))\right]>c(s),
\end{aligned}
$$

where the second equality follows from the fact that $W\left(s^{\prime}\right)=c\left(s^{\prime}\right)$ for all $s^{\prime} \in S^{a}$ and $W\left(s^{\prime}\right)=$ $\phi\left(s^{\prime}\right)$ for all $s^{\prime} \notin S^{a}$, the first inequality follows from the fact that $\phi\left(s^{\prime}\right) \leq \delta(\bar{c}+\bar{y}(\bar{c}-\delta \underline{c}))$ for all $s^{\prime}$, the second inequality follows since $W\left(s^{\prime}\right) \geq w\left(s^{\prime}\right)$ for all $s^{\prime}$ and the last inequality follows from (14). Therefore $W(s) \geq \phi(s)>c(s)$, so $s \notin S^{a}$.

Proposition 2 gives sufficient conditions for inefficiencies based on the primitives of the game: the level of optimism $y(s)$, the possible sizes of the surplus, and the value $w(s)$ of the single person optimal stopping problem (which itself depends on the transition matrix $P$ and the possible sizes of the surplus). By equation (14), inefficiencies are more likely to arise in settings in which players are optimistic about their bargaining power at states at which the value function $w(s)$ is larger; i.e., at states at which the surplus over which players are bargaining is larger. I stress however that, unlike equation (11) in Proposition 1, the conditions in Proposition 2 are not necessary for inefficient delays to arise in equilibrium; they are only sufficient.

\subsection{The benefits of optimism}

In this subsection, I study what happens to equilibrium payoffs when one of the agents becomes more optimistic. Let $p^{i} \in[0,1]^{|S|}$ be the vector specifying the beliefs of player $i$ about the probability with which she expects to make offers at each state $s \in S$. For

$\widetilde{p}^{i}, p^{i} \in[0,1]^{|S|}$, I write $\widetilde{p}^{i} \geq p^{i}$ whenever $\widetilde{p}_{s}^{i} \geq p_{s}^{i}$ for all $s \in S$, with at least one strict inequality.

Proposition 3 Assume $\widetilde{p}^{i} \geq p^{i}$, and let $V$ and $\widetilde{V}$ be the SPE payoffs when beliefs about the recognition process are given by $\left(p^{i}, p^{j}\right)$ and $\left(\widetilde{p}^{i}, p^{j}\right)$, respectively. Then, $\widetilde{V}_{i}(z) \geq V_{i}(z)$ and $\widetilde{V}_{j}(z) \leq V_{j}(z)$ for all $z \in Z$.

Proof. See Appendix A.2. 
Proposition 3 shows that there is a gain for a player who becomes more optimistic (and whose new level of optimism becomes common knowledge). For instance, if there was an initial stage prior to the game at which players could choose their beliefs, then by Proposition 3 it would be a weakly dominant strategy for both players to choose extremely optimistic beliefs (i.e., to choose $p_{s}^{i}=1$ for all $s \in S$ ).

\section{$5 \quad$ Frequent offers and efficiency}

In this section, I analyze the properties of equilibrium when players can make offers arbitrarily frequently. As usual, I can write the discounting between bargaining rounds as $\delta^{\Delta}=e^{-r \Delta}$ for some $r, \Delta>0$, where $r$ measures the rate of time preference and $\Delta$ is the time interval between bargaining rounds. The assumption that $\Delta>0$ captures the frictions in the bargaining process. The objective of this section is to study how the equilibrium outcome changes as these frictions disappear (i.e., as $\Delta \rightarrow 0$ ).

Fix an interval between offers $\Delta>0$. For each $s \in S$, the probability that $s_{t+\Delta}=s$ when $s_{t}=s$ is equal to $P_{s s}^{\Delta}=e^{-\lambda_{s} \Delta}$ for some $\infty>\lambda_{s} \geq 0 .{ }^{6}$ The probability that $s_{t+\Delta}=s^{\prime} \neq s$ when $s_{t}=s$ is equal to $P_{s s^{\prime}}^{\Delta}=\alpha_{s s^{\prime}}\left(1-e^{-\lambda_{s} \Delta}\right)$ for some $\alpha_{s s^{\prime}} \geq 0$, with $\sum_{s^{\prime} \neq s} \alpha_{s s^{\prime}}=1$. With this parametrization, the speed at which the Markov process moves remains roughly constant as I change the time between rounds $\Delta$. Let $P^{\Delta}=\left\{P_{s s^{\prime}}^{\Delta}\right\}_{s, s^{\prime} \in S}$ denote the transition matrix when the interval between bargaining rounds is $\Delta$. Finally, for any $f: S \rightarrow \mathbb{R}$, let $E_{s}^{\Delta}\left[f\left(s^{\prime}\right)\right]=\sum_{s^{\prime} \in S} P_{s s^{\prime}}^{\Delta} f\left(s^{\prime}\right)$ denote the expectation of $f\left(s_{t+\Delta}\right)$ conditional on $s_{t}=s$.

Let $S^{*}(\Delta):=\left\{s \in S: w^{\Delta}(s)=c(s)\right\}$, where $w^{\Delta}(s)=\max \left\{c(s), \delta^{\Delta} E_{s}^{\Delta}\left[w\left(s^{\prime}\right)\right]\right\}$. That is, $S^{*}(\Delta)$ is the set of states at which it is optimal to stop the process in the single person problem when the interval between periods is $\Delta$. Let $V^{\Delta}$ be the SPE payoffs when the interval between offers is $\Delta$. For each $s \in S$, let $W^{\Delta}(s)=V_{1}^{\Delta}(s, k)+V_{2}^{\Delta}(s, k)$. Hence, $S^{a}(\Delta):=\left\{s \in S: W^{\Delta}(s)=c(s)\right\}$ is the set of states at which there is agreement when the interval between bargaining rounds is $\Delta$. By Theorem $2, S^{a}(\Delta) \subseteq S^{*}(\Delta)$ for all $\Delta>0$. The main result of this section is that $S^{a}(\Delta)$ converges to $S^{*}(\Delta)$ as $\Delta \rightarrow 0$. In words, the outcome of the bargaining game converges to the efficient outcome when players can make offers arbitrarily frequently.

For any $\varepsilon>0$ and any interval between offers $\Delta$, let

$$
S_{\varepsilon}^{*}(\Delta):=\left\{s \in S: c(s) \geq \delta^{\Delta} E_{s}^{\Delta}\left[w\left(s^{\prime}\right)\right]+\varepsilon\right\} .
$$

\footnotetext{
${ }^{6}$ The restriction that $\lambda_{s}<\infty$ for all $s \in S$ implies that for any $\Delta>0$ there is positive probability that the state of the Markov process next period will be the same as the state today.
} 
That is, $S_{\varepsilon}^{*}(\Delta)$ is the set of states at which it is strictly optimal (by an amount weakly larger than $\varepsilon$ ) to stop the single person process and consume the surplus. Note that $S_{\varepsilon}^{*}(\Delta) \subseteq S_{\varepsilon^{\prime}}^{*}(\Delta)$ for any $\varepsilon>\varepsilon^{\prime} \geq 0$, and that $S_{0}^{*}(\Delta)=S^{*}(\Delta)$.

Theorem 3 For every $\varepsilon>0$, there exists $\bar{\Delta}(\varepsilon)>0$ such that $S_{\varepsilon}^{*}(\Delta) \subseteq S^{a}(\Delta) \subseteq S^{*}(\Delta)$ for all $\Delta<\bar{\Delta}(\varepsilon)$.

Proof. See Appendix A.3.

Theorem 3 shows that inefficiencies disappear when players can make offers arbitrarily frequently. In other words, this model can only deliver inefficient delays in cases in which there are non-negligible frictions in the bargaining process. In this sense, Theorem 3 extends the results of Yildiz (2003) to a stochastic bargaining game. As discussed in the Introduction, Yildiz (2003) showed that excessive optimism about the recognition process cannot be by itself a cause of inefficient delays. Similarly, in this paper's model inefficiencies vanish when players can make offers arbitrarily frequently.

As a way to illustrate the content in Theorem 3, consider a setting in which the surplus can take two possible values, $c(h)$ and $c(l)<c(h)$. Let $P_{l l}^{\Delta}=e^{-\lambda_{l} \Delta}$ and $P_{h h}^{\Delta}=e^{-\lambda_{h} \Delta}$, with $\lambda_{s} \in[0, \infty)$ for $s=h, l$. By equation (11) in Proposition 1, for any $\Delta>0$ the outcome of this stochastic bargaining game will be inefficient if and only if

$$
\begin{aligned}
& \frac{e^{-r \Delta}\left(1-e^{-\lambda_{l} \Delta}\right)(1+y(h)) c(h)}{\left(1-e^{-r \Delta} e^{-\lambda_{l} \Delta}\right)\left(1+e^{-r \Delta} y(h) e^{-\lambda_{h} \Delta}\right)+e^{-2 r \Delta}\left(1-e^{-\lambda_{l} \Delta}\right) y(h)\left(1-e^{-\lambda_{h} \Delta}\right)} \\
>c(l) \geq \frac{e^{-r \Delta}\left(1-e^{-\lambda_{l} \Delta}\right) c(h)}{1-e^{-r \Delta} e^{-\lambda_{l} \Delta}} &
\end{aligned}
$$

One can check that the left hand side and the right hand side of (15) both converge to $\lambda_{l} c(h) /\left(r+\lambda_{l}\right)$ as $\Delta \rightarrow 0$, so there is no room for inefficiencies when players can make offers arbitrarily frequently.

I assumed throughout this section that $\lambda_{s}<\infty$ for all $s \in S$, where $\lambda_{s}$ is such that $P_{s s}^{\Delta}=\operatorname{Pr}\left(s_{t+\Delta}=s \mid s_{t}=s\right)=e^{-\lambda_{s} \Delta}$. This restriction implies that there are no instantly transient states; that is, there are no states such that once the Markov process gets into them the probability of moving away from them in the next bargaining round is one, regardless of how short the interval between rounds is. The proof of Theorem 3 relies strongly on this restriction. Indeed, inefficient delays may persist even as $\Delta \rightarrow 0$ if $P_{s s}^{\Delta}=0$ for all $\Delta>0$ for some $s \in S$, as the following example shows.

Example 3 Suppose $S=\{h, l\}$, with $c(h)>c(l)$. Assume that $P_{h h}^{\Delta}=0$ for all $\Delta$ and 
$P_{l l}^{\Delta}=e^{-\lambda_{l} \Delta}$ for some $\lambda_{l} \in(0, \infty)$. By equation (11) in Proposition 1 , for any $\Delta>0$ the outcome of this stochastic bargaining game will be inefficient if and only if

$$
\frac{e^{-r \Delta}\left(1-e^{-\lambda_{l} \Delta}\right)(1+y(h)) c(h)}{\left(1-e^{-r \Delta} e^{-\lambda_{l} \Delta}\right)+e^{-2 r \Delta}\left(1-e^{-\lambda_{l} \Delta}\right) y(h)}>c(l) \geq \frac{e^{-r \Delta}\left(1-e^{-\lambda_{l} \Delta}\right) c(h)}{1-e^{-r \Delta} e^{-\lambda_{l} \Delta}} .
$$

The left-hand side of (16) converges to $\lambda_{l}(1+y(h)) c(h) /\left(r+\lambda_{l}(1+y(h))\right)$ as $\Delta \rightarrow 0$, while the right-hand side of (16) converges to $\lambda_{l} c(h) /\left(r+\lambda_{l}\right)$ as $\Delta \rightarrow 0$. Therefore, the limiting equilibrium outcome will display inefficiencies if and only if

$$
\frac{\lambda_{l}(1+y(h)) c(h)}{r+\lambda_{l}(1+y(h))}>c(l) \geq \frac{\lambda_{l} c(h)}{r+\lambda_{l}} .
$$

Note that there is a non-empty set of parameters for which these inequalities hold. Therefore, inefficient delays may persist in the continuous-time limit when some states are instantly transient.

To understand the intuition behind Theorem 3 and Example 3, consider first the case in which there are no instantly transient states (i.e., $0 \leq \lambda_{s}<\infty$ for all $s \in S$ ). In this case, the difference between the utility players get when they make offers and what they get when they don't (i.e., $\left.V_{i}^{\Delta}(s, i)-V_{i}^{\Delta}(s, j)\right)$ converges to zero as the time between periods goes to zero. Indeed, when $\lambda_{s}<\infty$ the probability that the state will be the same in the next bargaining round becomes arbitrarily close to one as $\Delta \rightarrow 0$. Furthermore, a short time between bargaining rounds also implies essentially no discounting from one round to the next. Since a new player is recognized to be proposer at each round, the cost in terms of discounting that the responder incurs by waiting until she becomes proposer converges to zero as $\Delta \rightarrow 0$, so $V_{i}^{\Delta}(s, i)-V_{i}^{\Delta}(s, j) \rightarrow 0$ as $\Delta \rightarrow 0$. In the continuous-time limit each player obtains the same payoffs when she proposer and when she is responder, so the differences in beliefs about the recognition process no longer have an impact on the sum of what the players expect to gain by delaying an agreement. As a result, efficiency is restored.

Consider next the case in which $P_{s s}^{\Delta}=0$ for all $\Delta>0$ for some $s \in S$ such that $c(s)>c\left(s^{\prime}\right)$ for all $s^{\prime} \neq s$ (as in Example 3). In this case, if the current state is $s$ the players know that with probability one the state will be different from $s$ in the next bargaining round, and that it may take a long time for the Markov process to come back to state $s$. Since $c(s)>c\left(s^{\prime}\right)$ for all $s^{\prime} \neq s$, the player who is making offers at state $s$ has a great deal of bargaining power, because if the other player rejects her offer the size of the surplus in the next bargaining round will be significantly smaller. Moreover, even if the time interval between offers is 
small, there might still be a substantial cost of delay in waiting for the Markov process to return to state $s$. In this case, the difference between the payoff a player gets at state $s$ when she makes offers and what she gets when she is responder (i.e., $\left.V_{i}^{\Delta}(s, i)-V_{i}^{\Delta}(s, j)\right)$ will remain bounded away from zero even as $\Delta \rightarrow 0$. Therefore, as Example 3 shows, in this case inefficiencies may persist even as $\Delta \rightarrow 0$.

\section{Conclusion}

This paper studies an infinite horizon bargaining game in which the size of the surplus follows a stochastic process and in which players might have optimistic beliefs about their future bargaining power. In a setup with a constant surplus, Yildiz (2003) showed that optimistic players will always come to an immediate agreement provided optimism is persistent and the number of bargaining rounds is large enough. In contrast, this paper shows that persistent optimism about bargaining power can generate potentially long lasting inefficient delays in settings in which players bargain over a stochastic surplus. The ultimate reason for this is that optimism about future bargaining power has more impact when the surplus is stochastic, since now players can be optimistic about their relative bargaining power at states at which the surplus is large.

However, this paper also shows that these inefficiencies can only occur when bargaining frictions are significant. As Theorem 3 shows, inefficiencies disappear when players can make offers arbitrarily frequently. The key reason behind this is that the right to make proposals becomes extremely transient when the time between bargaining rounds converges to zero, since a new player is selected to make offers at each round. This implies that the noninformational rent that a player obtains when she is proposer goes to zero when offers are frequent: in the continuous-time limit, players get the same payoff when they are making offers and when they are not. As a result, when offers are arbitrarily frequently optimism about future bargaining power no longer affects the sum of the players' continuation payoffs, and the outcome of the game becomes fully efficient.

The reasoning in the previous paragraph suggests that, in settings in which there is some persistence in the right to make proposals, optimism about bargaining power could potentially generate inefficient delays even when players can make offers arbitrarily frequently. The following example illustrates that this intuition is correct.

Example 4 Suppose $S=\{h, l\}$, with $c(h)=1>c(l)$. Assume further that $P_{h h}^{\Delta}=1$ for all $\Delta>0$ and that $P_{l l}^{\Delta}=e^{-\lambda_{l} \Delta}$ for some $\lambda_{l} \in(0, \infty)$. Let $\delta^{\Delta}=e^{-r \Delta}$ denote the common 
discount factor. At each round before agreement is reached, the player making offers last period retains the right to make offers with probability $q^{\Delta}=e^{-\gamma \Delta}$ (with $\gamma \in(0, \infty)$ ). With probability $1-q^{\Delta}$ natures selects a new player to be proposer. The parameter $\gamma$ measures the persistence of proposal power: $\gamma \rightarrow 0$ corresponds to the case in which proposal power is perfectly persistent, while $\gamma \rightarrow \infty$ corresponds to the case in which proposal power is transient. For simplicity, suppose that both players believe that they will be recognized to make offers with probability 1 every time nature selects a new proposer.

One can show that, for any $\Delta>0$, players will reach an immediate agreement in any subgame that starts at state $h$ : the proposer will obtain a payoff of $\bar{V}^{\Delta}=\left(1-\delta^{\Delta} q^{\Delta}\right) /(1-$ $\left.2 \delta^{\Delta} q^{\Delta}+\delta^{\Delta}\right)$ and the responder obtains $\underline{V}^{\Delta}=\delta^{\Delta}\left(1-q^{\Delta}\right) /\left(1-2 \delta^{\Delta} q^{\Delta}+\delta^{\Delta}\right){ }^{7}$ Let $V_{i}^{\Delta}(l, k)$ denote the payoff player $i$ gets from always delaying an agreement when the size of the surplus is $l$ and player $k$ is making offers:

$$
\begin{aligned}
& V_{i}^{\Delta}(l, i)=\delta^{\Delta}\left(\begin{array}{c}
P_{l l}^{\Delta}\left(q^{\Delta} V_{i}^{\Delta}(l, i)+\left(1-q^{\Delta}\right) V_{i}^{\Delta}(l, i)\right) \\
+\left(1-P_{l l}^{\Delta}\right)\left(q^{\Delta} \bar{V}+\left(1-q^{\Delta}\right) \bar{V}\right)
\end{array}\right), \\
& V_{j}^{\Delta}(l, i)=\delta^{\Delta}\left(\begin{array}{c}
P_{l l}^{\Delta}\left(q^{\Delta} V_{j}^{\Delta}(l, i)+\left(1-q^{\Delta}\right) V_{j}^{\Delta}(l, j)\right) \\
+\left(1-P_{l l}^{\Delta}\right)\left(q^{\Delta} \underline{V}+\left(1-q^{\Delta}\right) \bar{V}\right)
\end{array}\right) .
\end{aligned}
$$

Let $W^{\Delta}(l)=V_{1}^{\Delta}(l, 1)+V_{2}^{\Delta}(l, 1)=V_{1}^{\Delta}(l, 2)+V_{2}^{\Delta}(l, 2)$. Note that players will delay at state $l$ whenever $W^{\Delta}(l)>c(l)$, since in this case there is no agreement that can satisfy both players' expectations. Using (17) and (18), it follows that

$$
\begin{aligned}
W^{\Delta}(l)= & \frac{\delta^{\Delta} P_{l l}^{\Delta}\left(1-q^{\Delta}\right)}{1-\delta^{\Delta} P_{l l}^{\Delta}} \frac{\delta^{\Delta}\left(1-P_{l l}^{\Delta}\right) q^{\Delta}}{1-\delta^{\Delta} P_{l l}^{\Delta} q^{\Delta}}\left(\bar{V}^{\Delta}-\underline{V}_{\Delta}\right) \\
& +\frac{\delta^{\Delta}\left(1-P_{l l}^{\Delta}\right)}{1-\delta^{\Delta} P_{l l}^{\Delta}}\left(q^{\Delta}+\left(1-q^{\Delta}\right) 2 \bar{V}^{\Delta}\right) .
\end{aligned}
$$

In the continuous-time limit, it is efficient to consume the surplus at state $l$ if and only if $c(l) \geq \lambda_{l} /\left(r+\lambda_{l}\right)$. On the other hand, players will delay at state $l$ in the continuous-time limit if

$$
\lim _{\Delta \rightarrow 0} W^{\Delta}(l)=\frac{\lambda_{l} r \gamma}{\left(r+\lambda_{l}\right)(r+2 \gamma)\left(r+\gamma+\lambda_{l}\right)}+\frac{\lambda_{l}}{r+\lambda_{l}}>c(l) .
$$

Therefore, for any $\gamma \in(0, \infty)$ there is a non-empty set of parameters for which there will be inefficient delays even as players can make offers arbitrarily frequently.

\footnotetext{
${ }^{7}$ Indeed, when the size of the surplus is constant the model in this example is a special case of a model studied by Simsek and Yildiz (2009); and they show that in their more general model players will always come to an immediate agreement regardless of the level of optimism.
} 


\section{A Appendix}

\section{A.1 Proof of Theorem 1}

Let $F^{2}(Z)$ be the set of bounded functions on $Z$ taking values in $\mathbb{R}^{2}$. Let $\|\cdot\|$ denote the sup norm on $\mathbb{R}^{2}$. For any $f \in F^{2}(Z)$, let $\|f\|^{Z}=\sup \{\|f(z)\|: z \in Z\}$. For any $h: S \rightarrow \mathbb{R}$, let $E_{s}\left[h\left(s^{\prime}\right)\right]=\sum_{s^{\prime} \in S} P_{s s^{\prime}} h\left(s^{\prime}\right)$ denote the expectation of $h\left(s_{t+1}\right)$ conditional on $s_{t}=s$. Define the operator $A: F^{2}(Z) \rightarrow F^{2}(Z)$ as

$$
\begin{aligned}
& A_{i}(f)(s, i)=\max \left\{\begin{array}{c}
c(s)-\delta E_{s}\left[p_{s^{\prime}}^{j} f_{j}\left(s^{\prime}, j\right)+\left(1-p_{s^{\prime}}^{j}\right) f_{j}\left(s^{\prime}, i\right)\right], \\
\delta E_{s}\left[p_{s^{\prime}}^{i} f_{i}\left(s^{\prime}, i\right)+\left(1-p_{s^{\prime}}^{i}\right) f_{i}\left(s^{\prime}, j\right)\right]
\end{array}\right\} \\
& A_{i}(f)(s, j)=\delta E_{s}\left[p_{s^{\prime}}^{i} f_{i}\left(s^{\prime}, i\right)+\left(1-p_{s^{\prime}}^{i}\right) f_{i}\left(s^{\prime}, j\right)\right] .
\end{aligned}
$$

Lemma A1 The operator $A: F^{2}(Z) \rightarrow F^{2}(Z)$ is a contraction.

Proof. Let $f, g \in F^{2}(Z)$. For any state $(s, i) \in Z$,

$$
\begin{aligned}
\left|A_{j}(f)(s, i)-A_{j}(g)(s, i)\right| & =\left|\delta E_{s}\left[p_{s^{\prime}}^{j}\left(f_{j}\left(s^{\prime}, j\right)-g_{j}\left(s^{\prime}, j\right)\right)+\left(1-p_{s^{\prime}}^{j}\right)\left(f_{j}\left(s^{\prime}, i\right)-g_{j}\left(s^{\prime}, i\right)\right)\right]\right| \\
& \leq \delta E_{s}\left|\left(p_{s^{\prime}}^{j}\left(f_{j}\left(s^{\prime}, j\right)-g_{j}\left(s^{\prime}, j\right)\right)+\left(1-p_{s^{\prime}}^{j}\right)\left(f_{j}\left(s^{\prime}, i\right)-g_{j}\left(s^{\prime}, i\right)\right)\right)\right| \\
& \leq \delta\|f-g\|^{Z} .
\end{aligned}
$$

Next I show that $\left|A_{i}(f)(s, i)-A_{i}(g)(s, i)\right| \leq \delta\|f-g\|^{Z}$. Assume wlog that $A_{i}(f)(s, i) \geq$ $A_{i}(g)(s, i)$. There are two possible cases: (1) $A_{i}(f)(s, i)=c(s)-\delta E_{s}\left[p_{s^{\prime}}^{j} f_{j}\left(s^{\prime}, j\right)+(1-\right.$ $\left.\left.p_{s^{\prime}}^{j}\right) f_{j}\left(s^{\prime}, i\right)\right]$, or $(2) A_{i}(f)(s, i)=\delta E_{s}\left[p_{s^{\prime}}^{i} f_{i}\left(s^{\prime}, i\right)+\left(1-p_{s^{\prime}}^{i}\right) f_{i}\left(s^{\prime}, j\right)\right]$. In case $(1)$,

$$
\begin{aligned}
\left|A_{i}(f)(s, i)-A_{i}(g)(s, i)\right| & =\left|c(s)-\delta E_{s}\left[p_{s^{\prime}}^{j} f_{j}\left(s^{\prime}, j\right)+\left(1-p_{s^{\prime}}^{j}\right) f_{j}\left(s^{\prime}, i\right)\right]-A_{i}(g)(s, i)\right| \\
& \leq\left|\delta E_{s}\left[p_{s^{\prime}}^{j}\left(g_{j}\left(s^{\prime}, j\right)-f_{j}\left(s^{\prime}, j\right)\right)+\left(1-p_{s^{\prime}}^{j}\right)\left(g_{j}\left(s^{\prime}, i\right)-f_{j}\left(s^{\prime}, i\right)\right)\right]\right| \\
& \leq \delta\|f-g\|^{Z} .
\end{aligned}
$$

On the other hand, in case (2),

$$
\begin{aligned}
\left|A_{i}(f)(s, i)-A_{i}(g)(s, i)\right| & =\left|\delta E_{s}\left[p_{s^{\prime}}^{i} f_{i}\left(s^{\prime}, i\right)+\left(1-p_{s^{\prime}}^{i}\right) f_{i}\left(s^{\prime}, j\right)\right]-A_{i}(g)(s, i)\right| \\
& \leq\left|\delta E_{s}\left[p_{s^{\prime}}^{i}\left(f_{i}\left(s^{\prime}, i\right)-g_{i}\left(s^{\prime}, i\right)\right)+\left(1-p_{s^{\prime}}^{i}\right)\left(f_{i}\left(s^{\prime}, j\right)-g_{i}\left(s^{\prime}, j\right)\right)\right]\right| \\
& \leq \delta\|f-g\|^{Z} .
\end{aligned}
$$

Hence, $\|A(f)-A(g)\|^{Z} \leq \delta\|f-g\|^{Z}$, so $A$ is a contraction. 
For any pair $M, m \in F^{2}(Z)$ and for $j=1,2, i \neq j$, define

$$
H_{j}\left(M_{j}, m_{i}\right)(s, k)=\max \left\{c(s)-m_{i}(s, k), \delta E_{s}\left[p_{s^{\prime}}^{j} M_{j}\left(s^{\prime}, j\right)+\left(1-p_{s^{\prime}}^{j}\right) M_{j}\left(s^{\prime}, i\right)\right]\right\} .
$$

Define the operator $H: F^{2}(Z) \times F^{2}(Z) \rightarrow F^{2}(Z)$ as $H(M, m)=\left(H_{1}\left(M_{1}, m_{2}\right), H_{2}\left(M_{2}, m_{1}\right)\right)$.

Proof of Theorem 1. To prove Theorem 1, I start out assuming that the set of SPE payoffs is non-empty. At the end of the proof I show that the game indeed has a SPE.

Let $(u, \tau)$ be a SPE outcome and let $f_{i}(z)=E_{i}\left[\delta^{\tau} u_{i} \mid z_{0}=z\right]$ be the payoff that player $i$ gets from this SPE when the initial state is $z \in Z$. Let $\bar{M}=\left(\bar{M}_{1}, \bar{M}_{2}\right)$ and $\bar{m}=\left(\bar{m}_{1}, \bar{m}_{2}\right)$ denote the supremum and infimum SPE payoffs of players 1 and 2 (so that $\bar{M}_{i}(z) \geq f_{i}(z) \geq$ $\bar{m}_{i}(z)$ for all $\left.z \in Z, i=1,2\right)$. At any state $z=(s, i)$, player $j \neq i$ can guarantee herself a payoff of at least $A_{j}\left(\bar{m}_{j}, \bar{M}_{i}\right)(s, i)=\delta E_{s}\left[p_{s^{\prime}}^{j} \bar{m}_{j}\left(s^{\prime}, j\right)+\left(1-p_{s^{\prime}}^{j}\right) \bar{m}_{j}\left(s^{\prime}, i\right)\right]$ by rejecting the offer. Similarly, at state $z=(s, j)$ player $j$ 's payoff is at least as large as

$$
A_{j}\left(\bar{m}_{j}, \bar{M}_{i}\right)(s, j)=\max \left\{\begin{array}{c}
c(s)-\delta E_{s}\left[p_{s^{\prime}}^{i} \bar{M}_{i}\left(s^{\prime}, i\right)+\left(1-p_{s^{\prime}}^{i}\right) \bar{M}_{i}\left(s^{\prime}, j\right)\right], \\
\delta E_{s}\left[p_{s^{\prime}}^{j} \bar{m}_{j}\left(s^{\prime}, j\right)+\left(1-p_{s^{\prime}}^{j}\right) \bar{m}_{j}\left(s^{\prime}, i\right)\right]
\end{array}\right\}
$$

as player $i \neq j$ never rejects an offer of $\delta E_{s}\left[p_{s^{\prime}}^{i} \bar{M}_{i}\left(s^{\prime}, i\right)+\left(1-p_{s^{\prime}}^{i}\right) \bar{M}_{i}\left(s^{\prime}, j\right)\right]$. This implies that, for all $z \in Z, f_{j}(z) \geq A_{j}\left(\bar{m}_{j}, \bar{M}_{i}\right)(z)$ for $j=1,2, i \neq j$. For any pair $M, m \in F^{2}(Z)$ and for $j=1,2, i \neq j$, let $G_{j}(M, m)=A_{j}\left(m_{j}, M_{i}\right)$. Define the operator $G: F^{2}(Z) \times$ $F^{2}(Z) \rightarrow F^{2}(Z)$ as $G(M, m)=\left(G_{1}(M, m), G_{2}(M, m)\right)$.

Suppose next that the state is $(s, j)$. If player $j$ makes an offer that player $i$ accepts, then $f_{j}(s, j)+f_{i}(s, j) \leq c(s)$. Since $f_{i}(s, j) \geq \bar{m}_{i}(s, j)$, it follows that $f_{j}(s, j) \leq c(s)-\bar{m}_{i}(s, j)$. If player $j$ passes or if player $i$ does not accept her offer, then $f_{j}(s, j) \leq \delta E_{s}\left[p_{s^{\prime}}^{j} \bar{M}_{j}\left(s^{\prime}, j\right)+\right.$ $\left.\left(1-p_{s^{\prime}}^{j}\right) \bar{M}_{j}\left(s^{\prime}, i\right)\right]$, since the right hand side of this inequality is player $j$ 's highest possible continuation payoff at state $(s, j)$. On the other hand, $f_{j}(s, i) \leq \delta E_{s}\left[p_{s^{\prime}}^{j} \bar{M}_{j}\left(s^{\prime}, j\right)+(1-\right.$ $\left.\left.p_{s^{\prime}}^{j}\right) \bar{M}_{j}\left(s^{\prime}, i\right)\right]$ when the state is $(s, i)$, as player $j$ will always accept any offer that gives her this much. It then follows that $f_{j}(s, k) \leq H_{j}\left(\bar{M}_{j}, \bar{m}_{i}\right)(s, k)$ for $j=1,2, i \neq j$ (where $H(\cdot, \cdot)$ was defined above).

Next, let $M^{\prime}, M^{\prime \prime}$ and $m^{\prime}, m^{\prime \prime}$ (all bounded functions on $Z$ taking values on $\mathbb{R}^{2}$ ) be such that $M_{i}^{\prime}(z) \geq M_{i}^{\prime \prime}(z)$ for all $z \in Z, i=1,2$ and $m_{i}^{\prime}(z) \leq m_{i}^{\prime \prime}(z)$ for all $z \in Z, i=1,2$. One can check that this implies that $H_{i}\left(M^{\prime}, m^{\prime}\right)(z) \geq H_{i}\left(M^{\prime \prime}, m^{\prime \prime}\right)(z)$ for all $z \in Z, i=1,2$ and $G_{i}\left(M^{\prime}, m^{\prime}\right)(z) \leq G_{i}\left(M^{\prime \prime}, m^{\prime \prime}\right)(z)$ for all $z \in Z, i=1,2$. It what follows, for any pair $f, g \in F^{2}(Z)$ I will write $f \geq g$ if $f_{i}(z) \geq g_{i}(z)$ for all $z \in Z, i=1,2$. 
Define the sequences $\left\{M^{r}\right\}$ and $\left\{m^{r}\right\}$ as follows. Let $\left(M^{1}, m^{1}\right)=(\bar{M}, \bar{m})$, and for all $r \geq 2$ let $\left(M^{r}, m^{r}\right)=\left(H\left(M^{r-1}, m^{r-1}\right), G\left(M^{r-1}, m^{r-1}\right)\right)$. Note that $M^{2}=H\left(M^{1}, m^{1}\right) \geq M^{1}$ and $m^{2}=G\left(M^{1}, m^{1}\right) \leq m^{1}$. It follows then by induction and using our observation in the previous paragraph that $\left\{M^{r}\right\}$ is an increasing sequence and $\left\{m^{r}\right\}$ is a decreasing sequence. Moreover, it must be that $m^{r} \geq 0$ for all $r$ and $M^{r} \leq b$ for all $r$, where $b$ is such that $b \geq c(s)$ for all $s \in S$ (such a $b$ always exists, since $S$ is a finite set). Thus, both $\left\{M^{r}\right\}$ and $\left\{m^{r}\right\}$ are bounded and monotonic sequences, so there exists $M^{*}$ and $m^{*}$ such that $\left\{M^{r}\right\} \rightarrow M^{*}$ and $\left\{m^{r}\right\} \rightarrow m^{*}$. Finally, since both $H$ and $G$ are continuous functions it follows that $H\left(M^{*}, m^{*}\right)=M^{*}$ and $G\left(M^{*}, m^{*}\right)=m^{*}$. Therefore, $m^{*} \leq m^{1}=\bar{m} \leq \bar{M}=M^{1} \leq M^{*}$.

The next step is to show that $M_{i}^{*}=m_{i}^{*}$ for $i=1,2$. Since $m^{*} \leq \bar{m} \leq \bar{M} \leq M^{*}$, this will imply that $\bar{m}=\bar{M}$, so there are unique SPE payoffs. The strategy to prove this is to show that both $\left(M_{1}^{*}, m_{2}^{*}\right)$ and $\left(m_{1}^{*}, M_{2}^{*}\right)$ are fixed points of the operator $A$, and to note that by Lemma A1 the operator $A$ is a contraction and therefore has a unique fixed point.

Consider $\left(M_{1}^{*}, m_{2}^{*}\right)$. By the analysis above, $H_{1}\left(M_{1}^{*}, m_{2}^{*}\right)=M_{1}^{*}$ and $G_{2}\left(M^{*}, m^{*}\right)=m_{2}^{*}$. By definition of the operator $G, m_{2}^{*}=G_{2}\left(M^{*}, m^{*}\right)=A_{2}\left(m_{2}^{*}, M_{1}^{*}\right)$. Thus, to show that $\left(M_{1}^{*}, m_{2}^{*}\right)$ is a fixed point of $A$ it suffices to show that $A_{1}\left(M_{1}^{*}, m_{2}^{*}\right)=M_{1}^{*}$. To see this, consider first states $z=(s, 1)$ in which player 1 is the proposer. For those states it must be that

$$
m_{2}^{*}(s, 1)=A_{2}\left(m_{2}^{*}, M_{1}^{*}\right)(s, 1)=\delta E_{s}\left[p_{s^{\prime}}^{2} m_{2}^{*}\left(s^{\prime}, 2\right)+\left(1-p_{s^{\prime}}^{2}\right) m_{2}^{*}\left(s^{\prime}, 1\right)\right] .
$$

Equation (A.1) together with the definition of operator $A$ implies

$$
\begin{aligned}
A_{1}\left(M_{1}^{*}, m_{2}^{*}\right)(s, 1) & =\max \left\{c(s)-m_{2}^{*}(s, 1), \delta E_{s}\left[p_{s^{\prime}}^{1} M_{1}^{*}\left(s^{\prime}, 1\right)+\left(1-p_{s^{\prime}}^{1}\right) M_{1}^{*}\left(s^{\prime}, 2\right)\right]\right\} \\
& =H_{1}\left(M_{1}^{*}, m_{2}^{*}\right)(s, 1)=M_{1}^{*}(s, 1) .
\end{aligned}
$$

Consider next states $z=(s, 2)$. There are two possible cases. The first case is

$$
\begin{aligned}
m_{2}^{*}(s, 2) & =c(s)-\delta E_{s}\left[p_{s^{\prime}}^{1} M_{1}^{*}\left(s^{\prime}, 1\right)+\left(1-p_{s^{\prime}}^{1}\right) M_{1}^{*}\left(s^{\prime}, 2\right)\right] \\
& \geq \delta E_{s}\left[p_{s^{\prime}}^{2} m_{2}^{*}\left(s^{\prime}, 2\right)+\left(1-p_{s^{\prime}}^{2}\right) m_{2}^{*}\left(s^{\prime}, 1\right)\right] .
\end{aligned}
$$

The second case is one in which

$$
\begin{aligned}
m_{2}^{*}(s, 2) & =\delta E_{s}\left[p_{s^{\prime}}^{2} m_{2}^{*}\left(s^{\prime}, 2\right)+\left(1-p_{s^{\prime}}^{2}\right) m_{2}^{*}\left(s^{\prime}, 1\right)\right] \\
& >c(s)-\delta E_{s}\left[p_{s^{\prime}}^{1} M_{1}^{*}\left(s^{\prime}, 1\right)+\left(1-p_{s^{\prime}}^{1}\right) M_{1}^{*}\left(s^{\prime}, 2\right)\right] .
\end{aligned}
$$


In either case, $\delta E_{s}\left[p_{s^{\prime}}^{1} M_{1}^{*}\left(s^{\prime}, 1\right)+\left(1-p_{s^{\prime}}^{1}\right) M_{1}^{*}\left(s^{\prime}, 2\right)\right] \geq c(s)-m_{2}^{*}(s, 2)$. Therefore,

$$
\begin{aligned}
H_{1}\left(M_{1}^{*}, m_{2}^{*}\right)(s, 2) & =\max \left\{c(s)-m_{2}^{*}(s, 2), \delta E_{s}\left[p_{s^{\prime}}^{1} M_{1}^{*}\left(s^{\prime}, 1\right)+\left(1-p_{s^{\prime}}^{1}\right) M_{1}^{*}\left(s^{\prime}, 2\right)\right]\right\} \\
& =\delta E_{s}\left[p_{s^{\prime}}^{1} M_{1}^{*}\left(s^{\prime}, 1\right)+\left(1-p_{s^{\prime}}^{1}\right) M_{1}^{*}\left(s^{\prime}, 2\right)\right]=A_{1}\left(M_{1}^{*}, m_{1}^{*}\right)(s, 2) .
\end{aligned}
$$

Hence, $A\left(M_{1}^{*}, m_{2}^{*}\right)=\left(M_{1}^{*}, m_{2}^{*}\right)$. A symmetric argument shows that $A\left(m_{1}^{*}, M_{2}^{*}\right)=\left(m_{1}^{*}, M_{2}^{*}\right)$. Since $A$ is a contraction with a unique fixed point, it must be that $\left(M_{1}^{*}, m_{2}^{*}\right)=\left(m_{1}^{*}, M_{2}^{*}\right)$.

So far I showed that if the set of SPE is not empty, then all SPE must be payoff equivalent. Now I show that the set of SPE is indeed non-empty. To see this, let $V$ be such that $A(V)=V$. I first construct an anonymous stationary outcome $\left(S^{a} \times N, \eta\right)$ with payoffs equal to $V$. Define $\phi: S \rightarrow \mathbb{R}$ as in the main text, and let $S^{a}=\{s \in S: \phi(s) \leq c(s)\}$. Let $\eta(s, j)=V(s, j) \in C(s, j)$ for all $(s, j) \in S^{a} \times N$. One can check using standard dynamic programing arguments that the players' payoffs from outcome $\left(S^{a} \times N, \eta\right)$ are equal to $V$.

To show that $\left(S^{a} \times N, \eta\right)$ is the outcome induced by a SPE, consider the following strategy profile. At any state $z=(s, i)$, agent $i$ proposes division $\eta(z)=V(z)$ if $z \in S^{a} \times N$ and passes otherwise. Responder $j \neq i$ accepts any proposal which gives her a payoff of no less than $V_{j}(s, i)=\delta E_{s}\left(p_{s^{\prime}}^{j} V_{j}\left(s^{\prime}, j\right)+\left(1-p_{s^{\prime}}^{j}\right) V_{j}\left(s^{\prime}, i\right)\right)$, and rejects any proposal that gives her a lower payoff. Note that this strategy profile induces outcome $\left(S^{a} \times N, \eta\right)$. Moreover, no player can gain by unilaterally deviating from her strategy at any state $z \in Z$.

For $i=1,2$, let $\Gamma_{i}^{*}$ denote the set of strategies of player $i$ that survive iterated elimination of conditionally dominated strategies. Let $\Gamma^{*}=\Gamma_{1}^{*} \times \Gamma_{1}^{*}$. Since $\Gamma^{*}$ contains all the SPE and since the set of SPE is non-empty, it follows that $\Gamma^{*}$ is non-empty. The following result generalizes arguments in Yildiz (2003) to show that the unique SPE payoffs derived in Theorem 1 can also be derived via iterated elimination of conditionally dominated strategies.

Proposition A1 Let $\left(V_{1}, V_{2}\right)$ be the unique SPE payoffs derived in Theorem 1. Then, for $i=1,2$, for each $z \in Z$ and for each strategy profile in $\Gamma^{*}$, player $i$ 's payoffs at time $t$ with $z_{t}=z$ are equal to $V_{i}(z)$.

Proof. For period $t$ with $z_{t}=z$, let $\underline{V}_{i, t}(z)$ and $\bar{V}_{i, t}(z)$ be the infimum and supremum payoffs of player $i$ at time $t$, respectively, given that the set of remaining strategy profiles is $\Gamma^{*}$ (with the infimum and supremum taken over all possible histories of play and all strategies of player $j$ in $\Gamma_{j}^{*}$ ). To establish the claim, I will show that $\underline{V}_{i, t}(z)=\bar{V}_{i, t}(z)$. As a first step, note that the stationarity of the game implies that $\underline{V}_{i, t}(z)=\underline{V}_{i, s}(z)$ and $\bar{V}_{i, t}(z)=\bar{V}_{i, s}(z)$ for any $t$ and $s$ such that $z_{t}=z_{s}=z$. Hence, for every state $z$, let $\underline{V}_{i}(z)$ and $\bar{V}_{i}(z)$ be the 
infimum and supremum payoffs of player $i$ at any time $t$ with $z_{t}=z$, respectively, given that the set of remaining strategy profiles is $\Gamma^{*}$.

Since $\underline{V}_{i}\left(s^{\prime}, k\right)$ is the lowest payoff player $i$ expects to get at state $\left(s^{\prime}, k\right)$ (given that players use strategies in $\left.\Gamma^{*}\right)$, for any $\varepsilon>0$ there exists an iteration in the elimination process such that $i$ expects to get at any time $t+1$ with $z_{t+1}=\left(s^{\prime}, k\right)$ at least $\underline{V}_{i}\left(s^{\prime}, k\right)-\varepsilon / \delta$ for any remaining strategy profile and any history of play. Hence, at any time $t$ with $z_{t}=(s, k)$, any strategy that gives $i$ less than $\delta E_{s}\left[p_{s^{\prime}}^{i} \underline{V}_{i}\left(s^{\prime}, i\right)+\left(1-p_{s^{\prime}}^{i}\right) \underline{V}_{i}\left(s^{\prime}, j\right)\right]-\varepsilon$ against any strategy of $j$ that remains at that iteration is conditionally dominated by not reaching an agreement. Hence, such a strategy must be eliminated. This implies that $\underline{V}_{i}(s, k) \geq \delta E_{s}\left[p_{s^{\prime}}^{i} \underline{V}_{i}\left(s^{\prime}, i\right)+\right.$ $\left.\left(1-p_{s^{\prime}}^{i}\right) \underline{V}_{i}\left(s^{\prime}, j\right)\right]$. Moreover, at states $(s, i)$, for player $j$ rejecting an offer $u=\left(u_{1}, u_{2}\right)$ with $u_{j}>\delta E_{s}\left[p_{s^{\prime}}^{j} \bar{V}_{j}\left(s^{\prime}, j\right)+\left(1-p_{s^{\prime}}^{j}\right) \bar{V}_{j}\left(s^{\prime}, i\right)\right]$ is conditionally dominated by accepting such an offer. This in turn implies that $\underline{V}_{i}(s, i) \geq c(s)-\delta E_{s}\left[p_{s^{\prime}}^{j} \bar{V}_{j}\left(s^{\prime}, j\right)+\left(1-p_{s^{\prime}}^{j}\right) \bar{V}_{j}\left(s^{\prime}, i\right)\right]$. These two inequalities together imply that $\underline{V}_{i}(s, k) \geq A_{i}\left(\underline{V}_{i}, \bar{V}_{j}\right)$ for all $(s, k) \in S \times N$ (where the operator $A_{i}$ was defined at the beginning of Appendix A.1).

Next, I show that $\bar{V}_{i}(s, k) \leq H_{i}\left(\bar{V}_{i}, \underline{V}_{i}\right)(s, k)$ for all $(s, k) \in S \times N$ (where the operator $H_{i}$ was defined after the proof of Lemma A1). To see this, consider first states $(s, j)$. At such states, player $i$ will always accept any offer $u=\left(u_{1}, u_{2}\right)$ with $u_{i}>\delta E_{s}\left[p_{s^{\prime}}^{i} \bar{V}_{i}\left(s^{\prime}, i\right)+\right.$ $\left.\left(1-p_{s^{\prime}}^{i}\right) \bar{V}_{i}\left(s^{\prime}, j\right)\right]$. Hence, any conditionally undominated offer of player $j$ must give $i$ a payoff weakly lower than $\delta E_{s}\left[p_{s^{\prime}}^{i} \bar{V}_{i}\left(s^{\prime}, i\right)+\left(1-p_{s^{\prime}}^{i}\right) \bar{V}_{i}\left(s^{\prime}, j\right)\right]$. On the other hand, if player $j$ passes on his right to make an offer, or if she makes an offer than player $i$ rejects, then player $i$ 's payoff is again bounded above by $\delta E_{s}\left[p_{s^{\prime}}^{i} \bar{V}_{i}\left(s^{\prime}, i\right)+\left(1-p_{s^{\prime}}^{i}\right) \bar{V}_{i}\left(s^{\prime}, j\right)\right]$. Consider

next states $(s, i)$. At such states, accepting any offer that gives player $j$ less than $\underline{V}_{j}(s, i)$ is a conditionally dominated strategy for $j$. Hence, player $i$ cannot expect to get more than $\max \left\{c(s)-\underline{V}_{j}(s, i), \delta E_{s}\left[p_{s^{\prime}}^{i} \bar{V}_{i}\left(s^{\prime}, i\right)+\left(1-p_{s^{\prime}}^{i}\right) \bar{V}_{i}\left(s^{\prime}, j\right)\right]\right\}$. Combining all these arguments yields the desired inequality.

Finally, using arguments identical to those used to prove uniqueness of SPE payoffs in the proof of Theorem 1 , one can show that there exists $\underline{V}_{i}^{*}$ and $\bar{V}_{i}^{*}$ such that $\underline{V_{i}^{*}} \leq \underline{V_{i}} \leq \bar{V}_{i} \leq \bar{V}_{i}^{*}$, with $\left(\underline{V}_{1}^{*}, \bar{V}_{2}^{*}\right)$ and $\left(\bar{V}_{1}^{*}, \underline{V}_{2}^{*}\right)$ both fixed points of the operator $A$. Since $A$ has a unique fixed point, for $i=1,2$ it must be that $\underline{V}_{i}^{*}=\bar{V}_{i}^{*}$, and hence $\underline{V}_{i}=\bar{V}_{i}$.

\section{A.2 Proof of Proposition 3}

Lemma A2 Let $f, f^{\prime} \in F^{2}(Z)$ be such that $f_{j}^{\prime}(z) \leq f_{j}(z)$ and $f_{i}^{\prime}(z) \geq f_{i}(z)$ for all $z \in Z$. Then, $A_{i}\left(f^{\prime}\right)(z) \geq A_{i}(f)(z)$ and $A_{j}\left(f^{\prime}\right)(z) \leq A_{j}(f)(z)$ for all $z \in Z$. 
Proof. Let $f, f^{\prime} \in F^{2}(Z)$ be such that $f_{j}^{\prime}(z) \leq f_{j}(z)$ and $f_{i}^{\prime}(z) \geq f_{i}(z)$ for all $z \in Z$. For $z=(s, j)$, it follows immediately from the definition of operator $A$ that $A_{i}(f)(s, j) \leq$ $A_{i}\left(f^{\prime}\right)(s, j)$. Next, let $z=(s, i)$. In this case,

$$
\begin{aligned}
A_{i}\left(f^{\prime}\right)(s, i) & =\max \left\{\begin{array}{c}
c(s)-\delta E_{s}\left[p_{s^{\prime}}^{j} f_{j}^{\prime}\left(s^{\prime}, j\right)+\left(1-p_{s^{\prime}}^{j}\right) f_{j}^{\prime}\left(s^{\prime}, i\right)\right], \\
\delta E_{s}\left[p_{s^{\prime}}^{i} f_{i}^{\prime}\left(s^{\prime}, i\right)+\left(1-p_{s^{\prime}}^{i}\right) f_{i}^{\prime}\left(s^{\prime}, j\right)\right]
\end{array}\right\} \\
& \geq \max \left\{\begin{array}{c}
c(s)-\delta E_{s}\left[p_{s^{\prime}}^{j} f_{j}\left(s^{\prime}, j\right)+\left(1-p_{s^{\prime}}^{j}\right) f_{j}\left(s^{\prime}, i\right)\right], \\
\delta E_{s}\left[p_{s^{\prime}}^{i} f_{i}\left(s^{\prime}, i\right)+\left(1-p_{s^{\prime}}^{i}\right) f_{i}\left(s^{\prime}, j\right)\right]
\end{array}\right\}=A_{i}(f)(s, i) .
\end{aligned}
$$

Hence, $A_{i}\left(f^{\prime}\right)(z) \geq A_{i}(f)(z)$ for all $z \in Z$. Next, I show that $A_{j}\left(f^{\prime}\right)(z) \leq A_{j}(f)(z)$ for all $z \in Z$. If $z=(s, i)$, it then follows from the definition of operator $A$ that $A_{j}\left(f^{\prime}\right)(s, i) \leq$ $A_{j}(f)(s, i)$. Next, let $z=(s, j)$. In this case,

$$
\begin{aligned}
A_{j}\left(f^{\prime}\right)(s, j) & =\max \left\{\begin{array}{c}
c(s)-\delta E_{s}\left[p_{s^{\prime}}^{i} f_{i}^{\prime}\left(s^{\prime}, i\right)+\left(1-p_{s^{\prime}}^{i}\right) f_{i}^{\prime}\left(s^{\prime}, j\right)\right], \\
\delta E_{s}\left[p_{s^{\prime}}^{j} f_{j}^{\prime}\left(s^{\prime}, j\right)+\left(1-p_{s^{\prime}}^{j}\right) f_{j}^{\prime}\left(s^{\prime}, i\right)\right]
\end{array}\right\} \\
& \leq \max \left\{\begin{array}{c}
c(s)-\delta E_{s}\left[p_{s^{\prime}}^{i} f_{i}\left(s^{\prime}, i\right)+\left(1-p_{s^{\prime}}^{i}\right) f_{i}\left(s^{\prime}, j\right)\right], \\
\delta E_{s}\left[p_{s^{\prime}}^{j} f_{j}\left(s^{\prime}, j\right)+\left(1-p_{s^{\prime}}^{j}\right) f_{j}\left(s^{\prime}, i\right)\right]
\end{array}\right\}=A_{j}(f)(s, j) .
\end{aligned}
$$

Hence, $A_{j}\left(f^{\prime}\right)(z) \leq A_{j}(f)(z)$ for all $z \in Z$.

Proof of Proposition 3. Let $A: F^{2}(Z) \rightarrow F^{2}(Z)$ be the operator defined in the Appendix when beliefs are $\left(p^{i}, p^{j}\right)$ and let $\widetilde{A}: F^{2}(Z) \rightarrow F^{2}(Z)$ be the same operator when beliefs are $\left(\widetilde{p}^{i}, p^{j}\right)$. Thus, $A(V)=V$ and $\widetilde{A}(\widetilde{V})=\widetilde{V}$. Let $\left\{f^{r}\right\}$ be the sequence with $f^{1}=V$ and $f^{r}=\widetilde{A}\left(f^{r-1}\right)$ for all $r \geq 2$.

I first show that $f_{i}^{2}(z) \geq f_{i}^{1}(z)$ and $f_{j}^{2}(z) \leq f_{j}^{1}(z)$ for all $z \in Z$. To see this, take first $z=(s, i)$. Since $f^{2}=\widetilde{A}\left(f^{1}\right)$, then

$$
\begin{aligned}
f_{i}^{2}(s, i) & =\max \left\{\begin{array}{c}
c(s)-\delta E_{s}\left[p_{s^{\prime}}^{j} f_{j}^{1}\left(s^{\prime}, j\right)+\left(1-p_{s^{\prime}}^{j}\right) f_{j}^{1}\left(s^{\prime}, i\right)\right], \\
\delta E_{s}\left[\widehat{p}_{s^{\prime}}^{i} f_{i}^{1}\left(s^{\prime}, i\right)+\left(1-\widehat{p}_{s^{\prime}}^{i}\right) f_{i}^{1}\left(s^{\prime}, j\right)\right]
\end{array}\right\} \\
& \geq \max \left\{\begin{array}{c}
c(s)-\delta E_{s}\left[p_{s^{\prime}}^{j} f_{j}^{1}\left(s^{\prime}, j\right)+\left(1-p_{s^{\prime}}^{j}\right) f_{j}^{1}\left(s^{\prime}, i\right)\right], \\
\delta E_{s}\left[p_{s^{\prime}}^{i} f_{i}^{1}\left(s^{\prime}, i\right)+\left(1-p_{s^{\prime}}^{i}\right) f_{i}^{1}\left(s^{\prime}, j\right)\right]
\end{array}\right\}=f_{i}^{1}(s, i),
\end{aligned}
$$

where the inequality follows from the fact that $\widetilde{p}_{s^{\prime}}^{i} \geq p_{s^{\prime}}^{i}$ for all $s^{\prime} \in S$ and from the fact that $f_{i}^{1}\left(s^{\prime}, i\right)=V_{i}\left(s^{\prime}, i\right) \geq V_{i}\left(s^{\prime}, j\right)=f_{i}^{1}\left(s^{\prime}, j\right)$ for all $s^{\prime} \in S$. Moreover, $f_{j}^{2}(s, i)=$ $\delta E_{s}\left[p_{s^{\prime}}^{j} f_{j}^{1}\left(s^{\prime}, j\right)+\left(1-p_{s^{\prime}}^{j}\right) f_{j}^{1}\left(s^{\prime}, i\right)\right]=f_{j}^{1}(s, i)$. Next, I consider $z=(s, j)$. Since $f_{i}^{1}\left(s^{\prime}, i\right)=$ $V_{i}\left(s^{\prime}, i\right) \geq V_{i}\left(s^{\prime}, j\right)=f_{i}^{1}\left(s^{\prime}, j\right)$ for all $s^{\prime} \in S$ and since $\widetilde{p}_{s^{\prime}}^{i} \geq p_{s^{\prime}}^{i}$ for all $s^{\prime} \in S$, it must be that 


$$
\begin{aligned}
f_{i}^{2}(s, j)=\delta E_{s}\left[\widetilde{p}_{s^{\prime}}^{i} f_{i}^{1}\left(s^{\prime}, i\right)+\left(1-\widetilde{p}_{s^{\prime}}^{i}\right) f_{i}^{1}\left(s^{\prime}, j\right)\right] \geq f_{i}^{1}(s, j) . \text { On the other hand, } \\
f_{j}^{2}(s, j)=\max \left\{\begin{array}{c}
c(s)-\delta E_{s}\left[\widetilde{p}_{s^{\prime}}^{i} f_{i}^{1}\left(s^{\prime}, i\right)+\left(1-\widetilde{p}_{s^{\prime}}^{i}\right) f_{i}^{1}\left(s^{\prime}, j\right)\right], \\
\delta E_{s}\left[p_{s^{\prime}}^{i} f_{j}^{1}\left(s^{\prime}, j\right)+\left(1-p_{s^{\prime}}^{i}\right) f_{j}^{1}\left(s^{\prime}, i\right)\right]
\end{array}\right\} \\
\quad \leq \max \left\{\begin{array}{c}
c(s)-\delta E_{s}\left[p_{s^{\prime}}^{i} f_{i}^{1}\left(s^{\prime}, i\right)+\left(1-p_{s^{\prime}}^{i}\right) f_{i}^{1}\left(s^{\prime}, j\right)\right], \\
\delta E_{s}\left[p_{s^{\prime}}^{i} f_{j}^{1}\left(s^{\prime}, j\right)+\left(1-p_{s^{\prime}}^{i}\right) f_{j}^{1}\left(s^{\prime}, i\right)\right]
\end{array}\right\}=f_{j}^{1}(s, j),
\end{aligned}
$$

where the inequality follows from $\widetilde{p}_{s^{\prime}}^{i} \geq p_{s^{\prime}}^{i}$ for all $s^{\prime} \in S$ and from the fact that $f_{i}^{1}\left(s^{\prime}, i\right)=$ $V_{i}\left(s^{\prime}, i\right) \geq V_{i}\left(s^{\prime}, j\right)=f_{i}^{1}\left(s^{\prime}, j\right)$ for all $s^{\prime} \in S$. Thus, $f_{i}^{2}(z) \geq f_{i}^{1}(z)$ and $f_{j}^{2}(z) \leq f_{j}^{1}(z)$ for all $z \in Z$. By Lemma A2, it follows that $f_{i}^{r}(z) \geq f_{i}^{r-1}(z)$ and $f_{j}^{r}(z) \leq f_{j}^{r-1}(z)$ for all $z \in Z$ and for all $r \geq 1$. Finally, since $\widetilde{A}$ is a contraction and $\widetilde{V}$ is its unique fixed point, it must be that $\left\{f^{r}\right\} \rightarrow \widetilde{V}$. Thus, $\widetilde{V}_{i}(z) \geq V_{i}(z)$ and $\widetilde{V}_{j}(z) \leq V_{j}(z)$ for all $z \in Z$.

\section{A.3 Proof of Theorem 3}

I first show that $\left\|W^{\Delta}-w^{\Delta}\right\|^{S} \rightarrow 0$ as $\Delta \rightarrow 0$. Note that the operators $T_{\Delta}: F(S) \rightarrow F(S)$ and $T_{\Delta}^{\prime}: F(S) \rightarrow F(S)$ defined as

$$
\begin{aligned}
& T_{\Delta}(f)(s)=\max \left\{c(s), e^{-r \Delta} E_{s}^{\Delta}\left[f\left(s^{\prime}\right)\right]\right\} \\
& T_{\Delta}^{\prime}(f)(s)=\max \left\{c(s), e^{-r \Delta} E_{s}^{\Delta}\left[\widetilde{v}^{\Delta}\left(s^{\prime}\right)+f\left(s^{\prime}\right)\right]\right\},
\end{aligned}
$$

are both contractions with modulus $e^{-r \Delta}<1$, and that $w^{\Delta}$ and $W^{\Delta}$ are their respective unique fixed points. Moreover,

$$
\begin{aligned}
\left\|W^{\Delta}-w^{\Delta}\right\|^{S} & =\left\|T_{\Delta}^{\prime} W^{\Delta}-T_{\Delta} w^{\Delta}\right\|^{S} \leq\left\|T_{\Delta}^{\prime} W^{\Delta}-T_{\Delta}^{\prime} w^{\Delta}\right\|^{S}+\left\|T_{\Delta}^{\prime} w^{\Delta}-T_{\Delta} w^{\Delta}\right\|^{S} \\
& \leq e^{-r \Delta}\left\|W^{\Delta}-w^{\Delta}\right\|^{S}+\left\|T_{\Delta}^{\prime} w^{\Delta}-T_{\Delta} w^{\Delta}\right\|^{S}
\end{aligned}
$$

where the first inequality is an application of the triangle inequality and the second one follows from the fact that $T^{\prime}$ is a contraction with modulus $e^{-r \Delta}$. By equation (A.2),

$$
\left\|W^{\Delta}-w^{\Delta}\right\|^{S} \leq \frac{\left\|T_{\Delta}^{\prime} w^{\Delta}-T_{\Delta} w^{\Delta}\right\|^{S}}{1-e^{-r \Delta}} .
$$

By equation (A.3), in order to show that $\left\|W^{\Delta}-w^{\Delta}\right\|^{S} \rightarrow 0$ as $\Delta \rightarrow 0$ it suffices to show that $\left\|T_{\Delta}^{\prime} w^{\Delta}-T_{\Delta} w^{\Delta}\right\|^{S} /\left(1-e^{-r \Delta}\right) \rightarrow 0$ as $\Delta \rightarrow 0$. Towards proving this, note first that $T_{\Delta}^{\prime} w^{\Delta}(s) \geq T_{\Delta} w^{\Delta}(s) \geq c(s)$ for all $s \in S$. This implies that $\left|T_{\Delta}^{\prime} w^{\Delta}(s)-T_{\Delta} w^{\Delta}(s)\right|=0$ 
whenever $T_{\Delta}^{\prime} w^{\Delta}(s)=c(s)$. Moreover, note that $W^{\Delta}(s)=T_{\Delta}^{\prime} W^{\Delta}(s) \geq T_{\Delta}^{\prime} w^{\Delta}(s)$ for all $s \in S$. Since $c(s)=W^{\Delta}(s)$ for all $s \in S^{a}(\Delta)$, it follows that $T_{\Delta}^{\prime} w^{\Delta}(s)=c(s)$ for all $s \in S^{a}(\Delta)$. Therefore, $\left|T_{\Delta}^{\prime} w^{\Delta}(s)-T_{\Delta} w^{\Delta}(s)\right|=0$ for all $s \in S^{a}(\Delta)$.

Consider next states $s \notin S^{a}(\Delta)$ such that $T_{\Delta}^{\prime} w^{\Delta}(s)>c(s)$ (note that for any other state $s$ it must be that $\left.\left|T_{\Delta}^{\prime} w^{\Delta}(s)-T_{\Delta} w^{\Delta}(s)\right|=0\right)$. Let $s$ be such a state and note that

$$
\begin{aligned}
\left|T_{\Delta}^{\prime} w^{\Delta}(s)-T_{\Delta} w^{\Delta}(s)\right| & =e^{-r \Delta} E_{s}\left[\widetilde{v}^{\Delta}\left(s^{\prime}\right)+w^{\Delta}\left(s^{\prime}\right)\right]-T_{\Delta} w^{\Delta}(s) \\
& \leq e^{-r \Delta} E_{s}\left[\widetilde{v}^{\Delta}\left(s^{\prime}\right)\right]=e^{-r \Delta}\left(1-e^{-\lambda_{s} \Delta}\right) \sum_{s \neq s^{\prime}} \alpha_{s s^{\prime}} \widetilde{v}^{\Delta}\left(s^{\prime}\right)
\end{aligned}
$$

where the first inequality follows from the fact that $T w^{\Delta}(s) \geq e^{-r \Delta} E_{s}\left[w^{\Delta}\left(s^{\prime}\right)\right]$ and the last equality follows from the fact that $s \notin S^{a}(\Delta)$ and thus $\widetilde{v}^{\Delta}(s)=0$. Moreover, for all $s^{\prime} \in S^{a}(\Delta)$

$$
\begin{aligned}
\widetilde{v}^{\Delta}\left(s^{\prime}\right) & =y\left(s^{\prime}\right)\left(V_{1}^{\Delta}\left(s^{\prime}, 1\right)-V_{1}^{\Delta}\left(s^{\prime}, 2\right)\right) \leq y\left(s^{\prime}\right) V_{1}^{\Delta}\left(s^{\prime}, 1\right) \frac{1-e^{-r \Delta} e^{-\lambda_{s} \Delta}}{1-e^{-r \Delta} e^{-\lambda_{s} \Delta}\left(1-p_{s^{\prime}}^{1}\right)} \\
& \leq y\left(s^{\prime}\right) c\left(s^{\prime}\right) \frac{1-e^{-r \Delta} e^{-\lambda_{s^{\prime}} \Delta}}{1-e^{-r \Delta} e^{-\lambda_{s^{\prime}} \Delta}\left(1-p_{s^{\prime}}^{1}\right)},
\end{aligned}
$$

where the first inequality follows from $V_{1}^{\Delta}\left(s^{\prime}, 2\right) \geq e^{-r \Delta} e^{-\lambda_{s^{\prime}} \Delta}\left(p_{s^{\prime}}^{1} V_{1}^{\Delta}\left(s^{\prime}, 1\right)+\left(1-p_{s^{\prime}}^{1}\right) V_{1}^{\Delta}\left(s^{\prime}, 2\right)\right)$ and the second inequality follows from the fact that $V_{1}^{\Delta}\left(s^{\prime}, 1\right) \leq c\left(s^{\prime}\right)$ for all $s^{\prime} \in S^{a}(\Delta)$. Thus, for all $s \notin S^{a}(\Delta)$ it must be that

$$
\left|T_{\Delta}^{\prime} w^{\Delta}(s)-T_{\Delta} w^{\Delta}(s)\right| \leq e^{-r \Delta}\left(1-e^{-\lambda_{s} \Delta}\right) \sum_{s^{\prime} \neq s} \alpha_{s s^{\prime}} y\left(s^{\prime}\right) c\left(s^{\prime}\right)\left(\frac{1-e^{-r \Delta} e^{-\lambda_{s^{\prime}} \Delta}}{1-e^{-r \Delta} e^{-\lambda_{s^{\prime}} \Delta}\left(1-p_{s^{\prime}}^{1}\right)}\right) .
$$

Equations (A.3) and (A.5), the definition of $\|\cdot\|^{S}$ and the fact that $\left|T_{\Delta}^{\prime} w^{\Delta}(s)-T_{\Delta} w^{\Delta}(s)\right|=0$ for all $s \in S^{a}(\Delta)$ imply that

$$
\begin{aligned}
\left\|W^{\Delta}-w^{\Delta}\right\|^{S} & \leq \frac{e^{-r \Delta}}{1-e^{-r \Delta}} \max _{s \notin S^{a}(\Delta)}\left(\left(1-e^{-\lambda_{s} \Delta}\right) \sum_{s^{\prime} \neq s} \alpha_{s s^{\prime}} y\left(s^{\prime}\right) c\left(s^{\prime}\right)\left(\frac{1-e^{-r \Delta} e^{-\lambda_{s^{\prime}} \Delta}}{1-e^{-r \Delta} e^{-\lambda_{s^{\prime}} \Delta}\left(1-p_{s^{\prime}}^{1}\right)}\right)\right) \\
& \leq \frac{e^{-r \Delta}}{1-e^{-r \Delta}} \max _{s \in S}\left(\left(1-e^{-\lambda_{s} \Delta}\right) \sum_{s^{\prime} \neq s} \alpha_{s s^{\prime}} y\left(s^{\prime}\right) c\left(s^{\prime}\right)\left(\frac{1-e^{-r \Delta} e^{-\lambda_{s^{\prime}} \Delta}}{1-e^{-r \Delta} e^{-\lambda_{s^{\prime}} \Delta}\left(1-p_{s^{\prime}}^{1}\right)}\right)\right) .
\end{aligned}
$$

I now show that $\frac{e^{-r \Delta}\left(1-e^{-\lambda_{s} \Delta}\right)}{1-e^{-r \Delta}} y\left(s^{\prime}\right) c\left(s^{\prime}\right) \frac{1-e^{-r \Delta} e^{-\lambda_{s^{\prime}} \Delta}}{1-e^{-r \Delta} e^{-\lambda_{s^{\prime}}}\left(1-p_{s^{\prime}}^{1}\right)} \rightarrow 0$ as $\Delta \rightarrow 0$ for every $s, s^{\prime} \in$ $S$, which by the equation above implies that $\left\|W^{\Delta}-w^{\Delta}\right\|^{S} \rightarrow 0$ as $\Delta \rightarrow 0$. There are 
two cases to consider: (i) $p_{s^{\prime}}^{1}=0$ and (ii) $p_{s^{\prime}}^{1}>0$. In case (i) $y\left(s^{\prime}\right)=0$. Therefore, $\frac{e^{-r \Delta}\left(1-e^{-\lambda_{s} \Delta}\right)}{1-e^{-r \Delta}} y\left(s^{\prime}\right) c\left(s^{\prime}\right) \frac{1-e^{-r \Delta} e^{-\lambda_{s^{\prime}} \Delta}}{1-e^{-r \Delta} e^{-\lambda_{s^{\prime}} \Delta}\left(1-p_{s^{\prime}}^{1}\right)}=0$ for all $\Delta>0$. In case (ii), one can check that

$$
\lim _{\Delta \rightarrow 0} \frac{e^{-r \Delta}\left(1-e^{-\lambda_{s} \Delta}\right)}{1-e^{-r \Delta}} y\left(s^{\prime}\right) c\left(s^{\prime}\right)\left(\frac{1-e^{-r \Delta} e^{-\lambda_{s^{\prime}} \Delta}}{1-e^{-r \Delta} e^{-\lambda_{s^{\prime}} \Delta}\left(1-p_{s^{\prime}}^{1}\right)}\right)=0
$$

Thus, I conclude that $\left\|W^{\Delta}-w^{\Delta}\right\|^{S} \rightarrow 0$ as $\Delta \rightarrow 0$.

Finally, fix any $\varepsilon>0$. Since $\max _{s \in S} \widetilde{v}^{\Delta}(s) \rightarrow 0$ as $\Delta \rightarrow 0$ (see equation (A.4)) and $\left\|W^{\Delta}-w^{\Delta}\right\|^{S} \rightarrow 0$ as $\Delta \rightarrow 0$, there exists $\bar{\Delta}(\varepsilon)>0$ such that $\max _{s \in S} \widetilde{v}^{\Delta}(s) \leq \frac{\varepsilon}{2}$ and $\left\|W^{\Delta}-w^{\Delta}\right\|^{S} \leq \frac{\varepsilon}{2}$ for all $\Delta<\bar{\Delta}(\varepsilon)$. I will show that $S_{\varepsilon}^{*}(\Delta) \subseteq S^{a}(\Delta)$ for all $\Delta<\bar{\Delta}(\varepsilon)$. Take $\Delta<\bar{\Delta}(\varepsilon)$ and $\widetilde{s} \in S_{\varepsilon}^{*}(\Delta)$, so $w^{\Delta}(\widetilde{s})=c(\widetilde{s}) \geq \delta^{\Delta} E_{\widetilde{s}}\left[w^{\Delta}\left(s^{\prime}\right)\right]+\varepsilon$. Suppose by contradiction that $\widetilde{s} \notin S^{a}(\Delta)$, so

$$
W^{\Delta}(\widetilde{s})=\delta^{\Delta} E_{\widetilde{s}}\left[\widetilde{v}^{\Delta}\left(s^{\prime}\right)+W^{\Delta}\left(s^{\prime}\right)\right]>c(\widetilde{s}) .
$$

Since $\max _{s \in S} \widetilde{v}^{\Delta}(s) \leq \frac{\varepsilon}{2}$ and $\left\|W^{\Delta}-w^{\Delta}\right\|^{S} \leq \frac{\varepsilon}{2}$, it follows that

$$
\begin{aligned}
c(\widetilde{s}) & <\delta^{\Delta} E_{\widetilde{s}}\left[\widetilde{v}^{\Delta}\left(s^{\prime}\right)+W^{\Delta}\left(s^{\prime}\right)\right] \\
& \leq \delta^{\Delta} \frac{\varepsilon}{2}+\delta^{\Delta} E_{\widetilde{s}}\left[w^{\Delta}\left(s^{\prime}\right)+\frac{\varepsilon}{2}\right]<\varepsilon+\delta^{\Delta} E_{\widetilde{s}}\left[w^{\Delta}\left(s^{\prime}\right)\right],
\end{aligned}
$$

a contradiction to the fact that $\widetilde{s} \in S_{\varepsilon}^{*}(\Delta)$. Thus, it must be that $\widetilde{s} \in S^{a}(\Delta)$. Hence, $S_{\varepsilon}^{*}(\Delta) \subseteq S^{a}(\Delta) \subseteq S^{*}(\Delta)$.

\section{Acknowledgements}

I thank Faruk Gul for his encouragement and support and Dilip Abreu for helpful comments and suggestions. I also thank two anonymous referees, whose comments led to substantial improvements of this paper. All remaining errors are my own.

\section{References}

[1] Abreu, D., Gul, F., 2000. Bargaining and Reputation. Econometrica 68, 85-117.

[2] Ali, S.N.M., 2006. Waiting to Settle: Multilateral Bargaining with Subjective Biases. J. Econ. Theory 130, 109-137. 
[3] Avery, C., Zemsky, P., 1994. Option Values and Bargaining Delays. Games Econ. Behav. 7, 139-153.

[4] Babcock, L., Loewenstein, G., 1997. Explaining Bargaining Impasse: The Role of SelfServing Biases. J. Econ. Perspect. 11, 109-126.

[5] Compte, O., Jehiel, P., 2004. Gradualism in Bargaining and Contribution Games. Rev. Econ. Stud. 71, 975-1000.

[6] Cripps, M., 1998. Markov Bargaining Games. J. Econ. Dynam. Control 22, 341-355.

[7] Dekel, E., Fudenberg, D., Levine, D., 2004. Learning to Play Bayesian Games. Games Econ. Behav. 46, 282-303.

[8] Feinberg, Y., Skrzypacz, A., 2005. Uncertainty About Uncertainty and Delay in Bargaining. Econometrica 73, 69-91.

[9] Fershtman, C., Seidmann, D., 1993. Deadline Effects and Inefficient Delay in Bargaining with Endogenous Commitment. J. Econ. Theory 60, 306-321.

[10] Kennan, J., Wilson, R., 1993. Bargaining with Private Information. J. Econ. Lit. 31, $45-104$.

[11] Merlo, A., Wilson, C., 1995. A Stochastic Model of Sequential Bargaining with Complete Information. Econometrica 63, 371-399.

[12] Merlo, A., Wilson, C., 1998. Efficient Delays in a Stochastic Model of Bargaining. Econ. Theory 11, 39-55.

[13] Rubinstein, A., 1982. Perfect Equilibrium in a Bargaining Model. Econometrica 50, $97-110$.

[14] Simsek, A., Yildiz, M., 2009. Durable Bargaining Power and Stochastic Deadlines. Working paper.

[15] Yildiz, M., 2003. Bargaining Without a Common Prior-An Immediate Agreement Theorem. Econometrica 71, 793-811.

[16] Yildiz, M., 2004. Waiting to Persuade. Quart. J. Econ. 119, 223-248.

[17] Yildiz, M., 2011. Bargaining with Optimism. Annu. Rev. Econ. 3, 451-478. 